\title{
DIFFUSION APPROXIMATION AND COMPUTATION OF THE CRITICAL SIZE
}

\author{
BY
}

C. BARDOS, R. SANTOS AND R. SENTIS

\begin{abstract}
This paper is devoted to the mathematical definition of the extrapolation length which appears in the diffusion approximation. To obtain this result, we describe the spectral properties of the transport equation and we show how the diffusion approximation is related to the computation of the critical size. The paper also contains some simple numerical examples and some new results for the Milne problem.
\end{abstract}

Introduction. The computation of the critical size and the diffusion approximation for the transport equation have been closely related and this is due to the following facts. First, the computation of the critical size is much easier for the diffusion approximation than for the original transport equation. Second, for the critical size one can consider a host media $X={ }_{\eta} X_{0}$ with $X_{0}$ given and $\eta$ a positive number. The problem of the critical size is then reduced to the computation of the parameter $\eta$. It turns out that when the transport operator is almost conservative, the critical value of the parameter $\eta$ is large and it is exactly for this range of value that the diffusion approximation is accurate.

On the other hand the "physical" boundary condition for the diffusion approximation is of the form

$$
q(x)+l \frac{\partial q}{\partial \nu}(x)=0, \quad x \in \partial X
$$

( $q$ denotes the density of particles, $\partial q / \partial \nu$ is the derivative of $q$ according to the outward normal, and $l$ is a number which is called the extrapolation length). To the best of our knowledge there is no mathematical proof of the relevance of (1) for a general $X$; we will describe in $§ I V$ how to justify the introduction of the extrapolation length and we will give some numerical results $(\S \mathrm{V})$ in the very simple example of the slab, which show the importance of (1).

The mathematical tools that we use in this paper are the following:

1. The spectral theory for the transport operator.

2. The justification of the diffusion approximation with the introduction of a scaling factor $\varepsilon$, related to the mean free path.

3. The study of the asymptotic behaviour of the principal eigenvalue of the transport operator, as it was done by one of the authors (Sentis $[16,17])$.

Received by the editors July 15, 1983 and, in revised form, September 27, 1983.

1980 Mathematics Subject Classification. Primary 45K05: Secondary 45M05. 
Since we will use a scaling argument we will assume that the coefficients of the transport equation are independent of $x$ and that the collision function $f\left(v, v^{\prime}\right)$ is rotationally invariant. This will lead to explicit results.

We do not consider the most general case but the situation that we describe is rich enough. It contains the essential tools and will be easy to generalize to other cases.

I. Notation and spectral theory for the transport operator. The function $u(x, v, t)$ denotes the density of particles (neutrons) which at time $t$ are located at the point $x$ with the velocity $v, x$ belongs to a bounded convex open set $\mathbf{R}^{N}(N=1,2,3)$ with a smooth boundary $\partial X ; v$ belongs to the space of velocities $V$ which will denote a ball, a sphere or a spherical annulus of $\mathbf{R}^{N}$,

$$
V=\{0 \leqslant a \leqslant|v| \leqslant b<+\infty\}
$$

$V$ is provided with the canonical probability measure (denoted by $d v$ ).

We assume that the boundary is absorbing. This means that no neutron leaving the media will be able to come back. Since the boundary is smooth, the exterior normal $\nu(x)$ is defined everywhere and the boundary condition is then

$$
u(x, v, t)=0 \text { for } x \in \partial X, v \in V \text { and } \nu(x) \cdot v<0 .
$$

In the media, $u(x, v, t)$ satisfies the transport equation

$$
\frac{\partial u}{\partial t}+v \cdot \nabla u+\sigma\left(u-(1+\gamma) \int_{V} f\left(v, v^{\prime}\right) u\left(x, v^{\prime}, t\right) d v^{\prime}\right)=0
$$

$\sigma$ is a positive constant which describes the absorption of the media; $f\left(v, v^{\prime}\right)$ is the cross section function. We assume that $f$ is rotationally invariant, symetric with respect to $v$ and $v^{\prime}$, strictly positive and normalised:

$$
\begin{gathered}
f\left(R v, R v^{\prime}\right)=f\left(v, v^{\prime}\right) \quad \forall R \in O(V) ;^{1} \forall\left(v, v^{\prime}\right) \in V \times V, \\
f\left(v, v^{\prime}\right)=f\left(v^{\prime}, v\right), \quad 0<\pi_{l} \leqslant f\left(v, v^{\prime}\right) \leqslant \pi_{u}, \\
\int_{V} f\left(v, v^{\prime}\right) d v^{\prime}=1 .
\end{gathered}
$$

The rotation-invariance property is satisfied by any function $f$ which depends only on the modulus of $v$ and $v^{\prime}$ and of the angle of the vectors $v$ and $v^{\prime}$. With the relation (5), $\gamma$ denotes the distance of the state of the media to the conservative configuration, $\pi_{l}$ and $\pi_{u}$ are cosntants. We will denote by $K$ the integral operator

$$
K u=\int_{V} f\left(v, v^{\prime}\right) u\left(v^{\prime}\right) d v^{\prime}
$$

$K$ is a compact operator in any $L^{p}(V)(1 \leqslant p \leqslant \infty)$ and a selfadjoint operator in $L^{2}(V)$.

For any $p$ we introduce the unbounded operator $A_{p}$ (the letter $p$ will be omitted whenever it is not explicitly needed) which is defined by the formulas

$$
\begin{array}{r}
D\left(A_{p}\right)=\left\{u \in L^{p}(X \times V) \mid v \cdot \nabla u \in L^{p}(X \times V) ; u(x, v)=0\right. \\
\left.\forall(x, v) \in(\partial X \times V)_{-}\right\},
\end{array}
$$

${ }^{1} O(V)$ denotes the group of the rotations leaving invariant $S^{N-1}$. 
where $(\partial X \times V)_{-}=\{(x, v) \in \partial X \times V / \nu(x) \cdot v<0\}$,

$$
A_{p} u=-v \cdot \nabla u
$$

Therefore, one can write the transport equation as an abstract evolution equation according to the formula

$$
\partial u / \partial t=A_{p} u-\sigma u+\sigma(1+\gamma) K u .
$$

The operator $T_{p}=A_{p}-\sigma+\sigma(1+\gamma) K$ is the generator of a strongly continuous semigroup $e^{t T_{p}}$ in $L^{p}(X \times V)$ for $1 \leqslant p<\infty$. For $p=+\infty$ it defines a semigroup of bounded operators but it is not continuous with respect to $t$. (All these results are classical and can be found in any textbook on transport equations (cf. also Bardos [3] for a complete treatment of the boundary condition).) For the description of the spectra $\Sigma\left(T_{p}\right)$ in $L^{p}(X \times V)$ of the transport operator we introduce the number $\sigma^{*}$ given by the formula

$$
\sigma^{*}=\sigma \quad \text { if } 0 \in V, \quad \sigma^{*}=+\infty \quad \text { if } 0 \notin V,
$$

and we have the following

THEOREM 1. (i) The spectra $\Sigma\left(T_{p}\right)$ is contained in the half-plane $\operatorname{Re} \lambda \leqslant \gamma \sigma$.

(ii) For $p=2$, the half-plane $\operatorname{Re} \lambda \leqslant-\sigma^{*}$ belongs to the spectra of $T_{p}$.

(iii) $\Sigma^{\prime}=\Sigma\left(T_{p}\right) \cap\left\{\lambda / \operatorname{Re} \lambda>-\sigma^{*}\right\}$ is a set of eigenvalues of finite multiplicity (eventually empty) independent of $p$, with no accumulation point (except eventually on the axis $\operatorname{Re} \lambda=-\sigma^{*}$ ).

(iv) For $t>0$ one has

$$
\Sigma\left(e^{t T_{p}}\right) \cap\left\{z / \operatorname{Re} z>e^{-t \sigma^{*}}\right\}=e^{t \Sigma^{\prime}} .
$$

(v) When $\Sigma^{\prime}$ is not empty the number $\Lambda=\sup \left\{\operatorname{Re} \lambda \mid \lambda \in \Sigma^{\prime}\right\}$ is a simple eigenvalue of $T_{p}$ and the corresponding eigenvector is a function of constant sign which does not vanish outside the set $(\partial X \times V)$ and $\Lambda$ is the type of the semigroup $e^{t T_{p}}($ i.e. $)$,

$$
\Lambda=\lim _{t \rightarrow \infty}\left(\log \left|e^{t T_{p}}\right|\right) / t .
$$

(vi) For $X$ large enough (containing a ball of radius $R$ (big enough)) the set $\Sigma^{\prime}$ is not empty.

(vii) The type $\Lambda(X)$ is an increasing function of $X$. It is continuous with respect to the domain $X$ in the following sense: When the measure of the set $\left(X \cup X^{\prime}\right) \backslash\left(X \cap X^{\prime}\right)$ goes to zero, $\Lambda\left(X^{\prime}\right)$ converges to $\Lambda(X)$.

This theorem is now classical and we will only give some comments and some references for the different statements.

The point (i) is a direct consequence of the perturbation theory for strongly continuous semigroups (cf. Kato [10]).

(ii) was observed for the first time by Lehner and Wing [14] and proved in full generality by Albertoni and Montagnini [1].

(iii) For $\operatorname{Re} \lambda>-\sigma^{*}$ the operator $\lambda I-A_{p}+\sigma$ can be inverted and $\lambda$ belongs to the spectra of $T_{p}$ if and only if 1 belongs to the spectra of the operator $H_{\lambda, p}$ defined by

$$
H_{\lambda, p}=\left(\lambda I-A_{p}+\sigma\right)^{-1} \sigma(1+\gamma) K
$$


According to Vidav [19] for $\operatorname{Re} \lambda>-\sigma^{*}$ the mapping $\lambda \rightarrow H_{\lambda, p}^{2}$ is holomorphic in the space of compact operators. And the inverse of $1-H_{\lambda, p}$ is given, whenever it exists by the relation

$$
\left(1-H_{\lambda, p}\right)^{-1}=\left(1+H_{\lambda, p}\right)\left(1-H_{\lambda, p}^{2}\right)^{-1} .
$$

Therefore (iii) is a consequence of the Shmulyan theorem [18]. Since $\lambda \in \Sigma_{p}^{\prime}$ is characterised by the existence of a nonzero solution $u_{\lambda}^{p} \in L^{p}(X \times V)$ of the equation

$$
u_{\lambda}^{P}-H_{\lambda, p} u_{\lambda}^{P}=0
$$

we have $\Sigma_{p}^{\prime} \subset \Sigma_{q}^{\prime}$ for $p \geqslant q$. On the other hand, (14) yields

$$
u_{\lambda}^{1}=H_{\lambda, 1}^{N+1} u_{\lambda}^{1}, \quad u_{\lambda}^{1} \in L^{1}(X \times V) .
$$

With the regularising properties of the kernel $H_{\lambda}^{N+1}$ ( $N$ large enough) one can deduce from (15) that $u_{\lambda}^{1}$ belongs to $L^{\infty}(X \times V)$; therefore, $\Sigma_{1}^{\prime}=\Sigma_{\infty}^{\prime}$ and the proof of (iii) is complete (cf. Mokhtar Kharroubi [15] for the original proof and the details).

(iv) For any generator $T$ of a strongly continuous semigroup we have

$$
e^{t \Sigma(T)} \subset \Sigma\left(e^{t T}\right)
$$

and the difference $\Sigma\left(e^{t T}\right) \backslash e^{t \Sigma(T)}$ may contain zero and any point of the continuous spectra of $e^{t T}$. On the other hand, any nonzero eigenvalue of $e^{t T}$ belongs to $e^{t \Sigma(T)}$. It has been proved by Vidav [20] that $e^{t T}$ has no continuous spectra in the region $\left\{z|| z \mid>e^{-t \sigma^{*}}\right\}$; therefore, the relation (11) holds.

(v) Assume that $\Sigma^{\prime} \neq \varnothing$; then according to the weak Krein-Rutman theorem [12, Theorem 6.1] applied to the operator $H_{\lambda}$ in $L^{p}(X \times V)$ we know that $\Lambda$ is an eigenvalue of $T_{p}$, and from the relation (11) we conclude that $\Lambda$ is the type of the semigroup $e^{t T_{p}}$. From the weak Krein-Rutman theorem we conclude also that there exists, for $\Lambda$, at least one nonnegative eigenvector $u_{\Lambda}$.

The proof of the simplicity of the eigenvalue $\Lambda$ and of the strict positivity of $u_{\lambda}$ (outside the set $(\partial X \times V))$ is a consequence of the inequality (valid for any $(x, v) \in X \times V)$

$$
\left(H_{\lambda}\right)^{2} g(x, v) \geqslant\left[\iint g(x, v) d x d v\right] \cdot C\left[1-e^{-\pi_{l} d(x, \partial X) / b}\right],
$$

where $b$ and $C$ are positive constants (for the proof, which follows an idea of Bardos, see, for instance, [17]). The strict positivity of $\pi_{l}$ in (17) is compulsory for this last statement. In Mokhtar Kharroubi [15] one finds a counterexample with $\pi_{l}=0$ and a principal eigenvalue which is not simple.

(vi) To prove this point it suffices to show that the type of the semigroup $e^{t T}$ is greater than $-\sigma^{*}$. On the other hand, it is clear that for any positive data $\varphi$, the solution of the equation (9) with $u(x, v, 0)=\varphi(x, v)$ is greater than the solution of

$$
\frac{\partial u}{\partial t}=A u-\sigma u+\pi_{l} \sigma(1+\gamma) \int_{V} u\left(x, v^{\prime}\right) d v
$$

with the same initial data. 
Therefore, one has to show that there exists $\lambda>-\sigma$ and $u_{\lambda} \neq \equiv 0$ which is the solution of the equation

$$
\begin{gathered}
\lambda u_{\lambda}+v \nabla u_{\lambda}+\sigma u_{\lambda}-\pi_{l}(1+\gamma) \int_{V} u_{\lambda}\left(X, v^{\prime}\right) d v^{\prime}=0 \\
\left.u_{\lambda}(x, v)\right|_{(\partial X \times V)_{-}}=0 .
\end{gathered}
$$

Or with $\mu=(\lambda+\sigma)>0$ and $q(x)=\int_{V} u_{\lambda}\left(x, v^{\prime}\right) d v^{\prime}$,

$$
q-\left(\mu-A_{p}\right)^{-1} \pi_{l}(1+\gamma) q=0 .
$$

The integration over the velocity space gives the equivalent relation

$$
q(x)-\pi_{l}(1+\gamma) \int_{V} d v \int_{0}^{t(x, v)} \bar{e}^{\mu t} q(x-v t) d t=0 .
$$

In (21), $t(x, v)$ is defined by the relation $t(x, v)=\operatorname{Inf}\{t>0 \mid x-t v \in \partial X\}$. Now, we denote by $\delta$ the constant $\pi_{l}(1+\gamma)$ and we compute the operator $E_{\mu}$ defined by

$$
E_{\mu} q=\int_{V} d v \int_{0}^{t(x, v)} \bar{e}^{\mu t} q(x-v t) d t .
$$

When $V$ is a sphere of radius $\mathfrak{r}$ we have

$$
E_{\mu} q=\int_{X} \frac{1}{\mathrm{r}} \frac{\bar{e}^{\left|x-x^{\prime}\right| \mu / \mathrm{r}}}{\left|x-x^{\prime}\right|^{N-1}} q\left(x^{\prime}\right) d x^{\prime}
$$

and when $V$ is a spherical annulus we have

$$
E_{\mu} q=\int_{X} \frac{1}{\left|x-x^{\prime}\right|^{N-1}}\left(\int_{a}^{b} \frac{\bar{e}^{\left|x-x^{\prime}\right| \mu / \mathrm{r}}}{\mathrm{r}} d \mathrm{r}\right) q\left(x^{\prime}\right) d x^{\prime} .
$$

Now in both cases $E_{\mu}$ is a selfadjoint positive operator and the existence of a nonzero $u_{\lambda}$ is equivalent to the existence of a number $\mu>0$ such that $1 / \delta=$ $1 / \pi_{l}(1+\gamma)$ is an eigenvalue (or the principal eigenvalue) of $E_{\mu}$. Now the principal eigenvalue is equal to the $L^{2}$-norm of the operator $E_{\mu}$. The kernel of $E_{\mu}$ is a continuous decreasing function of $\mu$ going to zero when $\mu$ goes to infinity; therefore, the principal eigenvalue of $E_{\mu}, \rho\left(E_{\mu}\right)$, is also a decreasing continuous function of $\mu$ going to zero with $\mu$ going to infinity. We have only to show that for $X$ large enough (containing a ball of radius $R$ ) there exist a $\mu>0$ such that $\rho\left(E_{\mu}\right)$ is large enough (bigger than $\delta^{-1}=1 / \pi_{l}(1+\gamma)$ ).

We use the Rayleigh-Ritz principle with a test function $\varphi$ equal to 1 in a ball of radius $R$ contained in $X$ and equal to zero outside this ball. We denote also by $\bar{E}_{\mu}(|\cdot|)$ the kernel of the operator $E_{\mu}$. We have

$$
\begin{aligned}
\rho\left(\bar{E}_{\mu}\right) & \geqslant \frac{C_{1}}{R^{N}} \int_{|x|<R / 2} d x\left(\int_{\left|x^{\prime}\right|<R} \bar{E}_{\mu}\left(\left|x-x^{\prime}\right|\right) d x^{\prime}\right) \\
& \geqslant \frac{C_{1}}{R^{N}} \int_{|x|<R / 2} d x \int_{|y|<R / 2} \bar{E}_{\mu}(|y|) d y .^{2}
\end{aligned}
$$

\footnotetext{
${ }^{2}$ As usual $C_{1}, C_{2}, \ldots$ will denote several constants which depend only on the dimension of the space.
} 
In the case of the sphere ( $E_{\mu}$ given by (23)) we have

$$
\begin{aligned}
\rho\left(E_{\mu}\right) & \geqslant C_{2} \int_{|y|<R / 2} \frac{\bar{e}^{\mu|. y|}}{|y|^{N-1}} d y \\
& \geqslant \frac{C_{2}}{\mu} \int_{|y|<\mu R / 2} \frac{\bar{e}^{|y|}}{|y|^{N-1}} d y .
\end{aligned}
$$

Now we choose $\mu_{0}$ such that one has

$$
\frac{C_{2}}{\mu_{0}} \int_{|y|<1} \frac{\bar{e}^{|\cdot| y \mid}}{|y|^{N-1}} d y \geqslant \delta^{-1}=\left(\pi_{l}(1+\gamma)\right)^{-1}
$$

and with $R>2 / \mu_{0}$ we will obtain

$$
\begin{aligned}
\rho\left(E_{\mu}\right) & \geqslant \frac{C_{2}}{\mu_{0}} \int_{|y|<\mu_{0} R / 2} \frac{\bar{e}^{|y|}}{|y|^{N-1}} d y \\
& \geqslant \frac{C_{2}}{\mu_{0}} \int_{|y|<1} \frac{\bar{e}^{|y|}}{|y|^{N-1}} d y \geqslant \delta^{-1}
\end{aligned}
$$

and the proof of the existence of the eigenvalue $\Lambda(X)$, for $X$ large enough, is complete. The computation in the case of the spherical annulus ( $E_{\mu}$ given by (24)) can be done in a similar way; it is left to the reader. Some analogous computation, for more general cross sesction, and absorbtion functions can be found in Mokhtar Kharroubi [15].

Now let $X^{\prime} \supset X$ be two open bounded convex sets. We denote by $T_{X}$ and $T_{X^{\prime}}$ the corresponding transport operators (with identical absorbing coefficient $\sigma$ and identical cross section $\left.f\left(v, v^{\prime}\right)\right)$ is the two spaces $L^{p}(X \times V)$ and $L^{p}\left(X^{\prime} \times V\right)$; for any function $\varphi$ defined in $X \times V$ we denote by $\tilde{\varphi}$ its extension by zero in $X^{\prime} \times V$ :

$\tilde{\varphi}(x, v)=\varphi(x, v)$ if $x \in X$ and $\tilde{\varphi}(x, v)=0$ if $x \in X^{\prime} \backslash X$. And we denote also by $\left.\varphi\right|_{X}$ the restriction to $X$ of any function defined in $X^{\prime} \times V$. If $X$ is large enough the operator $T_{X}$ has a principal eigenvalue $\Lambda(X)$ and a corresponding positive eigenvector $\varphi(x, v)$; now $\tilde{\varphi}(x, v)$ is also a positive function and $\left(\left(e^{t T} X^{\prime}\right) \tilde{\varphi}\right)$ is also positive. By a comparison argument it is easy to show that one has, for every $(x, v) \in X \times V$,

$$
\left(\left(e^{t T_{X^{\prime}}}\right) \tilde{\varphi}\right)(x, v) \geqslant\left(e^{t T_{X}} \varphi\right)(x, v)=e^{t \Lambda(X)} \varphi(x, v) .
$$

This proves that the type of the semigroup is an increasing function of the domain. Finally, the continuity with respect to $X$ of the function $\Lambda(X)$ can be found in Mokhtar Kharroubi [15].

II. The diffusion approximation. We introduce in this section a parameter $\varepsilon$ and we will consider in $X \times V$ the transport equation

$$
\frac{\partial u_{\varepsilon}}{\partial t}+\frac{1}{\varepsilon} v \cdot \nabla u_{\varepsilon}+\sigma \frac{u_{\varepsilon}-\left(1+\varepsilon^{2} \gamma\right) K u_{\varepsilon}}{\varepsilon^{2}}=0
$$

with the usual absorbing boundary condition. The parameter $\varepsilon$ is a scaling factor and the reason why we choose their order of magnitude will become clear in $\S I V$, which is devoted to the computation of the critical size. Following Larsen and Keller [13] 
or Blankenship and Papanicolaou [5] we introduce a formal expansion of the solution $u_{\varepsilon}$ :

$$
u_{\varepsilon}(x, v, t)=u_{0}(x, v, t)+\varepsilon u_{1}(x, v, t)+\varepsilon^{2} u_{2}(x, v, t)+\varepsilon^{3} u_{3}(x, v, t)+\cdots .
$$

This leads to the relations

$$
\begin{gathered}
u_{0}-K u_{0}=0, \\
v \cdot \nabla u_{0}+\sigma\left(u_{1}-K u_{1}\right)=0, \\
\frac{\partial u_{0}}{\partial t}+v \cdot \nabla u_{1}+\sigma\left(u_{2}-K u_{2}\right)-\sigma \gamma K u_{0}=0 \\
\frac{\partial u_{n}}{\partial t}+v \cdot \nabla u_{n+1}+\sigma\left(u_{n+2}-K u_{n+2}\right)-\sigma \gamma K u_{n}=0 \quad \text { for } n \geqslant 1 .
\end{gathered}
$$

$K$ is a selfadjoint strictly positive operator; therefore, according to the HarrisKrein-Rutman Theorem (Harris [9, Chapter III]) its principal eigenvalue (in $L^{2}(V)$, for instance) is simple. From the relation

$$
\int_{V} f\left(v, v^{\prime}\right) d v^{\prime}=1 ; \quad f\left(v, v^{\prime}\right) \geqslant 0,
$$

we deduce that the principal eigenvalue is 1 and that the corresponding eigenvector is the function $1_{v}: v \rightarrow 1$. Therefore the kernel of the operator $I-K$ coincides with the space (of one dimension) spanned by the constant and therefore for any $g \in \operatorname{Ker}(I-K)^{\perp}$ there is a solution of the equation

$$
(I-K) u=g \text {. }
$$

Since $\operatorname{Ker}(I-K)^{\perp}$ is the space of the functions $g(v)$ with mean value zero, $\int_{V} g(v) d v=0$. For any $g$ with mean value zero there exits a unique function $u$, with mean value zero, which satisfies the equation $u-K u=g$. In particular, since we have $\int_{v} v_{i} d v=0$, we introduce the function $D_{i}(v)$ which is the solution of the problem

$$
D_{i}-K D_{i}(v)=v_{i}, \quad \int_{V} D_{i}(v) d v=0 .
$$

We denote by $D(v)$ the vector of components $D_{i}(v)(1 \leqslant i \leqslant N)$ and for the solution of (33) we have the expression

$$
u_{1}(x, v, t)=-\frac{D(v)}{\sigma} \cdot \nabla u_{0}(x, t)+W_{1}(x, t),
$$

where $W_{1}(x, t)$ is a function independent of $v$.

The function $u_{2}(x, v, t)$ is given by the relation (34) and this equation will admit a solution if and only if

$$
\int\left(\frac{\partial u_{0}}{\partial t}+v \nabla u_{1}-\sigma \gamma K u_{0}\right) d v=0
$$

that is to say,

$$
\frac{\partial u_{0}}{\partial t}-\frac{1}{\sigma} \int_{V} v_{i} \frac{\partial}{\partial x_{i}}\left(D_{j}(v) \frac{\partial u_{0}}{\partial x_{j}}\right) d v-\sigma \gamma u_{0}=0 .
$$


In (38) appears a second order operator $\mathscr{D}$ which is defined by the expression

$$
u_{0} \rightarrow-\frac{1}{\sigma}\left(\int_{V} v_{i} D_{j} d v\right) \frac{\partial}{\partial x_{i}} \frac{\partial u_{0}}{\partial x_{j}} .
$$

With only the hypothesis $0<\pi_{l} \leqslant f\left(v, v^{\prime}\right) \leqslant \pi_{u}$, one can show that the quadratic form defined on $\mathbf{R}^{n}$ by the formula

$$
q(\xi)=\sum_{i j} \int_{V} v_{i} D_{j}(v) \xi_{i} \xi_{j} d v
$$

is positive nondegneerate. Therefore $\mathscr{D}$ is a second order elliptic operator. On the other hand, with the rotation-invariance property one can obtain a more explicit expression for this quadratic form and for the functions $D_{i}(v)$. Indeed, for any rotation $R \in O(V)$ we have

$$
R v=D(R v)-\int_{V} f\left(R v, v^{\prime}\right) D\left(v^{\prime}\right) d v^{\prime} .
$$

In the integral of the right-hand side of (40) we make the change of variable $v^{\prime}=R v^{\prime \prime}$ and obtain

$$
\begin{aligned}
R v & =D(R v)-\int_{V} f\left(R v, R v^{\prime \prime}\right) D\left(R v^{\prime \prime}\right) d v^{\prime \prime} \\
& =D(R v)-\int_{V} f\left(v, v^{\prime}\right) D\left(R v^{\prime}\right) d v^{\prime}
\end{aligned}
$$

(by the rotation-invariance of $f\left(v, v^{\prime}\right)$ ). Therefore we have

$$
D(R v)=R D(v) \text {. }
$$

This leads to the relation

$$
\begin{aligned}
q(R \xi) & =\sum_{i j} \int_{V} v_{i} D_{j}(v)(R \xi)_{i}(R \xi)_{j} d v \\
& =\int_{V}(v \cdot R \xi)(D(v) \cdot R(\xi)) d v \\
& =\int_{V}\left(R^{*} v \cdot \xi\right)\left(R^{*} D(v) \cdot \xi\right) d v \\
& =\int_{V}\left(R^{*} v \cdot \xi\right)\left(D\left(R^{*} v\right) \cdot \xi\right) d v \\
& =\int_{V}\left(v^{\prime} \cdot \xi\right)\left(D\left(v^{\prime}\right) \cdot \xi\right) d v^{\prime}
\end{aligned}
$$

(with $v^{\prime}=R^{*} v$ ). From the formula (43) we deduce that $q(\xi)$ is rotation-invariant and we have

$$
q(\xi)=e_{0} \sum_{i} \xi i^{2}
$$

This implies the relation

$$
\int_{V} v_{i} D_{j}(v) d v=e_{0} \delta_{i j}
$$


Equation (38) is then the diffusion equation

$$
\frac{\partial u_{0}}{\partial t}-k_{0} \nabla u_{0}-\sigma \gamma u_{0}=0
$$

where $k_{0}$ is a positive constant given by the formula

$$
k_{0}=e_{0} / \sigma \text {. }
$$

REMARK 1 . The integration, with respect to $v$, of equation (30) gives

$$
\frac{\partial q_{\varepsilon}}{\partial t}+\frac{1}{\varepsilon} \nabla \cdot \mathscr{T}_{\varepsilon}-\sigma \gamma q_{\varepsilon}=0
$$

with $q_{\varepsilon}(x, t)=\int_{V} u_{\varepsilon}(x, v, t) d v$ and $\mathscr{T}_{\varepsilon}=\int_{V} v \cdot u_{\varepsilon}(x, v, t) d v$, and it is natural to deduce by "physical arguments" that $\mathscr{T}_{\varepsilon}$, which is a current, is approximately proportional to the gradient of the charge $q_{\varepsilon}$. Therefore the above computation appears to be a rigourous proof of these arguments.

On the other hand, with the maximum principle one can obtain, for the solution of the inhomogenous transport equation

$$
\begin{gathered}
\frac{\partial w_{\varepsilon}}{\partial t}+\frac{1}{\varepsilon} v \cdot \nabla w_{\varepsilon}+\frac{\sigma}{\varepsilon^{2}}\left(I-\left(1+\varepsilon^{2} \gamma\right) K\right) w_{\varepsilon}=h_{\varepsilon}, \\
\left.w_{\varepsilon}(x, v, t)\right|_{(\partial X \times V)_{-}}=g_{\varepsilon}, \quad w_{\varepsilon}(x, v, 0)=\varphi_{\varepsilon},
\end{gathered}
$$

the following uniform (with respect to $\varepsilon$ ) a priori estimate: for any $(x, v, t) \in X \times V$ $\times \mathbf{R}_{+}$we have

$$
\begin{aligned}
\left|w_{\varepsilon}(x, v, t)\right| \leqslant & e^{\sigma \gamma t} \sup _{(x, v)}\left|\varphi_{\varepsilon}(x, v)\right| \\
& +e^{\sigma \gamma t}\left\{\sup _{(x, v, s) \in X \times V \times[0, t]}\left|h_{\varepsilon}(x, v, s)\right|\right. \\
& \left.+\sup _{(x, v, s) \in(\partial X \times V)_{-} \times[0, t]}\left|g_{\varepsilon}(x, v, s)\right|\right\} .
\end{aligned}
$$

With this inequality and the formal asymptotic expansion, one can prove the following

THEOREM 2. We assume that the initial data $u(x, v, 0)$ is independent of $v$ and we denote by $u_{\varepsilon}(x, v, t)$ and $u_{0}(x, t)$ the solutions of the transport equation

$$
\begin{gathered}
\frac{\partial u_{\varepsilon}}{\partial t}+\frac{1}{\varepsilon} v \cdot \nabla u_{\varepsilon}+\frac{\sigma}{\varepsilon^{2}}\left(I-\left(1+\varepsilon^{2} \gamma\right) K\right) u_{\varepsilon}=0, \\
\left.u_{\varepsilon}(x, v, t)\right|_{(\partial X \times V)_{-}}=0
\end{gathered}
$$

and of the diffusion equation, with the Dirichlet boundary condition,

$$
\frac{\partial u_{0}}{\partial t}-k_{0} \Delta u_{0}-\sigma \gamma u_{0}=0,\left.\quad u_{0}(\cdot, t)\right|_{\partial x}=0
$$

with the same initial data $u(x, v, 0)=u(x, 0)$. Then we have the uniform estimate

$$
\left|u_{\varepsilon}(x, v, t)-u_{0}(x, t)\right| \leqslant \varepsilon e^{\gamma t} C\left(u_{0}\right) .
$$


The proof of this theorem can be found in Blankenship and Papanicolaou [5]. One introduces the function

$$
w_{\varepsilon}(x, v, t)=u_{\varepsilon}(x, v, t)-u_{0}(x, t)-\varepsilon\left(\frac{-D(v)}{\sigma} \nabla u_{0}+w(x, t)\right)-\varepsilon^{2} u_{2}(x, v, t),
$$

where $w(x, t)$ is arbitrary and $u_{2}(x, v, t)$ is a solution of (34); then one can ue (50) and the fact that on $(\partial X \times V)$ _one has

$$
\begin{aligned}
w_{\varepsilon}(x, v, t) & =u_{\varepsilon}(x, v, t)-u_{0}(X, t)+\varepsilon\left(-\frac{D(v)}{\sigma} \nabla u_{0}+w(x, t)\right)-\varepsilon^{2} u_{2}(x, v, t) \\
& =\varepsilon\left(-\frac{D(v)}{\sigma} \cdot \nabla u_{0}+w(x, t)\right)+O\left(\varepsilon^{2}\right) .
\end{aligned}
$$

One of the main consequences of Theorem 2 is the fact that the operator $T_{\varepsilon}=A / \varepsilon$ $-\left(\sigma / \varepsilon^{2}\right)\left(I-\left(1+\varepsilon^{2} \gamma\right) K\right)$ converges to the operator $k_{0} \Delta+\sigma \gamma$ with the Dirichlet boundary condition in the following sense:

Let $\pi$ be the projection operator from $L^{2}(X \times V)$ on $L^{2}(X)$ defined by

$$
\pi f(x)=\int_{V} f(x, v) d v .
$$

Then for any $\alpha \geqslant 0$, we have the strong convergence in the space of the bounded operators on $L^{2}(X \times V)$

$$
\left(T_{\varepsilon}-\alpha\right)^{-1} \rightarrow\left(k_{0} \Delta_{D}+\sigma \gamma-\alpha\right)^{-1} \pi
$$

and one can prove

TheOREM 3 (SENTIS [17]). When $\varepsilon$ goes to 0 , the principal eigenvalue ${ }^{3} \Lambda_{\varepsilon}$ of the operator $T_{\varepsilon}$ converges to the principal eigenvalue $\Lambda_{d}$ of the operator $\left(k_{0} \Delta_{D}+\sigma \gamma\right)$ with the Dirichlet boundary condition.

As it will appear below, the error between $\Lambda_{\varepsilon}$ and $\Lambda_{d}$ is of the order of $\varepsilon$ and this is mainly due to the choice of the Dirichlet boundary condition for the diffusion operator, an improvement of the error will lead to the introduction of the extrapolation length.

Since we have $\Sigma\left(T_{\varepsilon}\right) \subset\{\lambda \mid \operatorname{Re} \lambda \leqslant \sigma \gamma\}$ and $\Sigma\left(k_{0} \Delta_{D}+\sigma \gamma\right) \subset\{\lambda \mid \lambda \leqslant \sigma \gamma\}$, we will introduce a positive constant $C>\sigma \gamma$ and study the order of the error between $\omega_{\varepsilon}=\Lambda_{\varepsilon}-C$ and $\omega_{d}=\Lambda_{d}-C$. The principal eigenvalue $\omega_{\varepsilon}$ of $T_{\varepsilon}-C$ is also the principal eigenvalue of its adjoint $\left(T_{\varepsilon}^{*}-C\right)$ which is simple. Let $\Psi_{\varepsilon}$ be the eigenvalue of $\left(T_{\varepsilon}^{*}-C\right)$ corresponding to $\omega_{\varepsilon}$ and normalized by

$$
\left\|\Psi_{\varepsilon}\right\|_{L^{2}(X \times V)}=1, \quad \Psi_{\varepsilon} \geqslant 0 .
$$

Following Sentis [17] we denote by $\Phi$ the principal eigenfunction of $k_{0} \Delta_{D}+\sigma \gamma-C$ normalized by $\|\phi\|_{L^{2}(X)}=1, \phi \geqslant 0$, and we introduce the solution of the equation

$$
\left(T_{\varepsilon}-C\right) z_{\varepsilon}=\omega_{d} \Phi ; \quad z_{\varepsilon} \in D\left(T_{\varepsilon}\right) .
$$

\footnotetext{
${ }^{3} \mathrm{~A}$ real eigenvalue of an operator is said to be its principal eigenvalue if it is greater than the real part of any spectral value.
} 
Now we have ${ }^{4}$

$$
\omega_{d}\left\langle\Phi, \Psi_{\varepsilon}\right\rangle=\left\langle\left(T_{\varepsilon}-C\right) z_{\varepsilon}, \Psi_{\varepsilon}\right\rangle=\left\langle z_{\varepsilon},\left(T_{\varepsilon}-C\right)^{*} \Psi_{\varepsilon}\right\rangle=\omega_{\varepsilon}\left\langle z_{\varepsilon}, \Psi_{\varepsilon}\right\rangle .
$$

From (56) we deduce the relation

$$
\omega_{d}\left\langle\Phi, \Psi_{\varepsilon}\right\rangle=\omega_{\varepsilon}\left\langle z_{\varepsilon}-\Phi, \Psi_{\varepsilon}\right\rangle+\omega_{\varepsilon}\left\langle\Phi, \Psi_{\varepsilon}\right\rangle
$$

and the relation

$$
\omega_{\varepsilon}-\omega_{d}=\omega_{\varepsilon}\left\langle z_{\varepsilon}-\Phi, \Psi_{\varepsilon}\right\rangle /\left\langle\Phi, \Psi_{\varepsilon}\right\rangle .
$$

One can show (cf. Sentis [17]) that $\Psi_{\varepsilon}$ converges to $\Phi$ in $L^{2}(X \times V)$ and since these functions are choosen of norm 1 , we have

$$
\lim _{\varepsilon \rightarrow 0} \frac{1}{\varepsilon}\left(\omega_{\varepsilon}-\omega_{d}\right)=\omega_{d} \lim _{\varepsilon \rightarrow 0} \frac{\left\langle z_{\varepsilon}-\Phi, \Psi_{\varepsilon}\right\rangle}{\varepsilon}
$$

Therefore the last step is the study of the limit in $L^{2}(X \times V)$ of the expression $\left(Z_{\varepsilon}-\Phi\right) / \varepsilon$ and at this point will appear a boundary layer and a corrector related to this boundary layer which will introduce the extrapolation length.

III. The construction of the corrector for the stationary solution and for the principal eigenvalue. Since we are concerned only by the eigenvalue of the operator $T_{\varepsilon}$ it will be sufficient to consider the stationary solution of the transport equation. Keeping in mind that the spectra of $T_{\varepsilon}$ lies in the half-space $\operatorname{Re} \lambda \leqslant \sigma \gamma$, we introduce a constant $C>\sigma \gamma$ and consider the solution $u_{\varepsilon}$ of the equation

$$
\begin{gathered}
C u_{\varepsilon}+\frac{1}{\varepsilon} v \cdot \nabla u+\frac{\sigma}{\varepsilon^{2}}\left(I-\left(1+\varepsilon^{2} \gamma\right) K\right) u_{\varepsilon}=g, \\
\left.u_{\varepsilon}(x, V)\right|_{(\partial X \times V)_{-}}=0 .
\end{gathered}
$$

We assume that the right-hand side of (56) is a function $g(x)$ independent of $v$ and smooth. With the usual expansion

$$
u_{\varepsilon}=u_{0}+\varepsilon u_{1}+\varepsilon^{2} u_{2}+\varepsilon^{3} u_{3}+h_{\varepsilon}
$$

we obtain the equations

$$
\begin{gathered}
u_{0}-K u_{0}=0, \\
v \cdot \nabla u_{0}+\sigma(I-K) u_{1}=0, \\
C u_{0}+v \cdot \nabla u_{1}+\sigma(I-K) u_{2}-\sigma \gamma K u_{0}=g, \\
C u_{1}+v \cdot \nabla u_{2}+\sigma(I-K) u_{3}-\sigma \gamma K u_{1}=0 .
\end{gathered}
$$

With the same argument as in the evolution equations described in §II, we deduce that $u_{0}$ is the solution of the Dirichlet elliptic equation

$$
(C-\gamma \sigma) u_{0}-\kappa_{0} \Delta u_{0}=g,\left.\quad u_{0}\right|_{\partial X}=0
$$

and that $u_{1}$ is given by the relation

$$
u_{1}(x, v)=-\frac{D(v)}{\sigma} \cdot \nabla u_{0}+w
$$

with $w$ being a function depending only on $x \in X$.

\footnotetext{
${ }^{4}\langle\cdot, \cdot\rangle$ denotes the scalar product on $L^{2}(X \times V)$.
} 
Now $u_{2}$ is given by the relation

$$
\sigma(I-K) u_{2}=g+(\sigma \gamma-C) u_{0}+\frac{1}{\sigma} v \cdot \nabla\left(D \cdot \nabla u_{0}\right)-v \cdot \nabla w
$$

The relation (64) and the compatibility condition (66) implies that $u_{2}$ is the sum of the function $-D(v) / \sigma \cdot \nabla w$ and of the solution $h(x, v)$ of the equation

$$
(I-K) h=\frac{1}{\sigma} v \cdot \nabla\left(D \cdot \nabla u_{0}\right)-k_{0} \Delta u_{0} .
$$

The right-hand side of (69) is an even function of $v$ and thanks to (36) we see that $h$ is also an even function of $v$. Equation (65) can be written as

$$
\begin{aligned}
\sigma(I-K) u_{3}= & -(C-\sigma \gamma)\left(-\frac{D(v)}{\sigma} \cdot \nabla u_{0}+w\right) \\
& -\sigma \gamma(I-K)\left(-\frac{D}{\sigma} \nabla u_{0}+w\right)-v \nabla\left(h-\frac{D}{\sigma} \nabla w\right),
\end{aligned}
$$

and for the solvability condition of the problem (70) we obtain

$$
(C-\sigma \gamma) w-k_{0} \Delta w=0 .
$$

Therefore, $w$ satisfies the same equation as $u_{0}$ but, up to now, no boundary condition is prescribed on $w$. With any $w$ satisfying (71) one can construct a solution $u_{3}$ of (70) and therefore, for the function

$$
\mathrm{r}_{\varepsilon}=u_{\varepsilon}-u_{0}-\varepsilon\left(-D(v) / \sigma \cdot \nabla u_{0}+w\right)-\varepsilon^{2} u_{2}-\varepsilon^{3} u_{3},
$$

we have the equation

$$
\begin{aligned}
& C \mathrm{r}_{\varepsilon}+\frac{1}{\varepsilon} \cdot v \nabla \mathrm{r}_{\varepsilon}+\frac{\sigma}{\varepsilon^{2}}(I-K) \mathrm{r}_{\varepsilon}=\varepsilon^{2} \theta(x, v), \\
& \mathrm{r}_{\varepsilon} l_{(\partial X \times V)_{-}}=\varepsilon\left(\frac{D(v)}{\sigma} \cdot \nabla u_{0}-w\right)+\varepsilon^{2} \delta(x, v) .
\end{aligned}
$$

$u_{0}$ is a smooth function which is zero on $\partial X$; therefore all the tangential derivatives of $u_{0}$ on $\partial X$ are all also zero, and the second equation of (72) turns out to be the equation

$$
\left.\mathrm{r}_{\varepsilon}\right|_{(\partial X \times V)_{-}}=\varepsilon\left(\frac{(D(v) \cdot \nu)}{\sigma} \frac{\partial u_{0}}{\partial \nu}-w\right)+\varepsilon^{2} \delta(x, v) .
$$

In (72), $\theta(x, v)$ and $\delta(x, v)$ denote two smooth functions independent of $\varepsilon$. We observe that the dominant factor comes from the boundary term and is of order $\varepsilon$. So in order to obtain an approximation of $u_{\varepsilon}$ of order $\varepsilon^{2}$ we have to build a boundary layer term. Since $\partial X$ is smooth and compact we assume the existence of a number $\delta>0$ such that the variable $\hat{x} \in \partial X ; y=(x-\hat{x}) \nu(\hat{x})$ gives a set of coordinates for the layer

$$
\{x \in X, d(x, \partial X) \leqslant \delta\}
$$


Let $\eta=y / \varepsilon=(x-\hat{x}) / \varepsilon \cdot \nu(\hat{x})$ and we consider (following Bensoussan, Lions and Papanicolaou [4]) the boundary layer equation (in the variables $\eta$ and $v, \hat{x}$ being only a parameter)

$$
\begin{gathered}
v \cdot \nu(\hat{x}) \frac{\partial b}{\partial \eta}+\sigma(I-K)=0 \text { in } \mathbf{R}^{-} \times V \\
b(0, v, \hat{x})=\frac{1}{\sigma} D(v) \nu(\hat{x}) \frac{\partial u_{0}}{\partial \nu}(\hat{x}) \quad \text { for } v \cdot \nu(\hat{x})<0 .
\end{gathered}
$$

This problem is the classical Milne problem. In the present case it is not an easy problem because it corresponds to the conservative case which implies that 0 is in the spectra of the operator

$$
T=v \cdot \nu \frac{\partial}{\partial \eta}+\sigma(I-K)
$$

defined in $L^{2}\left(\mathbf{R}^{-} \times V\right)$ with the domain

$$
D(T)=\left\{u \in L^{2}\left(\mathbf{R}^{-} \times V\right) \mid v \cdot \nu \frac{\partial u}{\partial \eta} \in L^{2}\left(\mathbf{R}^{-} \times V\right), u(0, v)=0 \text { for } v \cdot \nu<0\right\} .
$$

The main theorems concerning this problem can be found in some explicit cases in the classical books on transport theory (cf. Chandrasekhar [7]); in the general situation they are proved by Bensoussan, Lions and Papanicolaou [4]. The method of [4] relies on probabilistic tools. In Appendix 2 we will prove these results using only the tools of fundamental functional analysis. The main results (which are proved in Appendix 2) are summarized in the following (where $V_{-}=\left\{v \in V \mid v_{N}<0\right\}$.

THEOREM 4. (i) For any function $\varphi \in L^{\infty}\left(V_{-}\right)$there is a unique bounded solution $u \in L^{\infty}\left(\mathbf{R}_{-} \times V\right)$ of the problem

$$
v_{N} \frac{\partial u}{\partial n}+\sigma(I-K) u=0, \quad u(0, v)=\varphi(v) \quad \text { for } v \in V_{-} .
$$

(ii) When $\eta$ goes to $-\infty, u(\eta, v)$ converges exponentially fast to a constant $G(\varphi)$.

(iii) The mapping $\varphi \rightarrow G(\varphi)$ is a linear continuous mapping from $L^{\infty}\left(V_{-}\right)$to $\mathbf{R}$.

(iv) When $\varphi$ is positive, $u$ and also $G(\varphi)$ are positive.

(v) Let $D_{N}(v)$ be the component of $D(v)$ in the Nth direction. Then $G\left(D_{N}\right)=-L$ is a negative constant.

Now for the function $w(x)$, which is a solution of (71), we request the boundary condition

$$
\left.w\right|_{\partial X}=\frac{1}{\sigma} G\left(D_{N}\right) \frac{\partial u_{0}}{\partial \nu} .
$$

(71) and (76) define a classical Dirichlet boundary value problem which has a unqiue well-defined solution $w$. Then for

$$
\chi(\hat{x}, \eta, v)=b(\eta, v, \hat{x})-w(\hat{x})
$$


we have

$$
\begin{aligned}
& v \cdot \nu(\hat{x}) \frac{\partial \chi}{\partial \eta}+\sigma(I-K) \chi=0 \text { in } \mathbf{R}_{-} \times V, \\
& \chi(\hat{x}, 0, v)=\frac{1}{\sigma} D(v) \cdot \nu \frac{\partial u_{0}}{\partial \nu}-w(\hat{x}) \text { for } v \cdot \nu(\hat{x})<0, \\
& \chi(x, \eta, v) \rightarrow 0 \text { exponentially fast for } \eta \rightarrow-\infty .
\end{aligned}
$$

The last condition of (78) shows that $\chi$ is a boundary layer term. For

$$
s_{\varepsilon}=u_{\varepsilon}-\left(u_{0}+\varepsilon\left(\frac{-D(v)}{\sigma} \cdot \nabla u_{0}+w\right)+\varepsilon^{2} u_{2}+\varepsilon^{3} u_{\varepsilon}+\varepsilon \chi\left(\hat{x}, \frac{x-\hat{x}}{\varphi} \cdot \nu(\hat{x}), v\right)\right)
$$

we have the relations

$$
\begin{gathered}
c s_{\varepsilon}+\frac{1}{\varepsilon} v \cdot \nabla s_{\varepsilon}+\frac{\sigma}{\varepsilon^{2}}(I-K) s_{\varepsilon}=\varepsilon^{2} \theta_{\varepsilon}(x, v), \\
\left.s_{\varepsilon}\right|_{(\partial X \times V)_{-}}=\varepsilon^{2} \delta(x, v) .
\end{gathered}
$$

In (79), $\delta$ is the smooth function which appears in (72). Since $(\partial / \partial \eta) \chi(\hat{x}, \eta, v)$ converges to zero exponentially fast, when $\eta$ goes to $-\infty$, we see that, for any compact set $X^{\prime} \subset X$, we have

$$
\sup _{x \in X^{\prime}} \sup _{v \in V}\left|\theta_{\varepsilon}(x, v)\right| \leqslant C \quad \text { (independent of } \varepsilon \text { ). }
$$

Therefore, for any $p(1 \leqslant p<\infty)$ we have the estimate

$$
\left\|s_{\varepsilon}\right\|_{L^{p}(X \times V)} \leqslant \varepsilon^{2} C_{p},
$$

where $C_{p}$ denotes a constant depending on $p$ but independent of $\varepsilon$, and from (79) and (80) we can deduce the following theorem which is an explicit version of the results of Bensoussan, Lions and Papanicolaou [4].

Theorem 5. Assume that $g$ is a smooth function defined on $X$ and let $u_{\varepsilon}$ be the solution of the transport equation

$$
\begin{gathered}
c u_{\varepsilon}+\frac{1}{\varepsilon} v \cdot \nabla u_{\varepsilon}+\frac{\sigma}{\varepsilon^{2}}\left(u_{\varepsilon}-\left(1+\varepsilon^{2} \gamma\right) K u_{\varepsilon}\right)=g(x) \quad \text { in } X \times V, \\
\left.u_{\varepsilon}\right|_{(\partial X \times V)_{-}}=0 ;
\end{gathered}
$$

then in any $L^{p}(X \times V)$ space $(1 \leqslant p<\infty)$ we have the estimate

$$
\left\|u_{\varepsilon}-\left(u_{0}+\varepsilon\left(\frac{-D(v)}{\sigma} \cdot \nabla u_{0}+w\right)\right)\right\|_{L^{p}(X \times V)} \leqslant \varepsilon^{2} C_{p} .
$$

The constant $C_{p}$ depends on $p$ but is independent of $\varepsilon$ and the functions $u_{0}$ and $w$ are the solution of the two Dirichlet elliptic boundary value problems

$$
\begin{gathered}
(c-\sigma \gamma) u_{0}-k_{0} \Delta u_{0}=g,\left.\quad u_{0}\right|_{\partial X}=0, \\
(c-\sigma \gamma) w-k_{0} \Delta w=0,\left.\quad w\right|_{\partial X}=-\frac{L}{\sigma} \frac{\partial u_{0}}{\partial \nu} .
\end{gathered}
$$


REMARK 2. Since we have $\int_{V} D(v) \nabla w d v=0$, an approximation up to the order two in $\varepsilon$ of the density $\int_{V} u_{\varepsilon}(x, v) d v$ is given by the function $\tilde{u}_{\varepsilon}=u_{0}+\varepsilon w$.

REMARK 3. The function $\tilde{u}_{\varepsilon}$ satisfies the elliptic equation

$$
(c-\sigma \gamma) \tilde{u}_{\varepsilon}-k_{0} \Delta \tilde{u}_{\varepsilon}=0 \text { in } X
$$

with the boundary condition

$$
\tilde{u}_{\varepsilon}+\varepsilon \frac{L}{\sigma} \frac{\partial \tilde{u}_{\varepsilon}}{\partial \nu}=\varepsilon^{2} \frac{L}{\sigma} \frac{\partial w}{\partial \nu} \quad \text { on } \partial X .
$$

Since $L$ is positive, the problem (85) (86) (for $c \geqslant \sigma \gamma$ ) is always well posed. The corresponding selfadjoint operator is defined by the coercive quadratic form on the space $H^{1}(X)$,

$$
a(\varphi, \Psi)=k_{0} \int_{X} \nabla \varphi \cdot \nabla \Psi d x+(c-\sigma \gamma) \int_{X} \varphi \Psi d x+\frac{k_{0} \sigma}{\varepsilon L} \int_{\partial X} \varphi \Psi d x .
$$

We can now state the following

Proposition 1. Let $g$ be a smooth function on $X$ and $u_{\varepsilon}$ be the solution of the equation

$$
\begin{gathered}
c u_{\varepsilon}+\frac{1}{\varepsilon} v \cdot \nabla u_{\varepsilon}+\frac{\sigma}{\varepsilon^{2}}\left(I-\left(1+\varepsilon^{2} \gamma\right) K\right) u_{\varepsilon}=g, \\
\left.u_{\varepsilon}(x, v)\right|_{(\partial X \times V)_{-}}=0
\end{gathered}
$$

and let $\hat{u}_{\varepsilon}$ be the solution of the equation

$$
\begin{gathered}
(c-\sigma \gamma) \hat{u}_{\varepsilon}-k_{0} \Delta \hat{u}_{\varepsilon}=g \quad \text { in } X, \\
\hat{u}_{\varepsilon}+\varepsilon \frac{L}{\sigma} \frac{\partial \hat{u}_{\varepsilon}}{\partial \nu}=0 \quad \text { on } \partial X .
\end{gathered}
$$

Then for any $p(1 \leqslant p<\infty)$ with $C_{p}$ depending on $p$ but not on $\varepsilon$ we have the estimate

$$
\left\|\int_{V} u_{\varepsilon}(x, v) d v-\hat{u}_{\varepsilon}\right\|_{L^{p}(X)} \leqslant \varepsilon^{2} C_{p} .
$$

The proof of this proposition is a consequence of $(85),(86)$ and the equations

$$
\begin{aligned}
& \left((c-\sigma \gamma)-k_{0} \Delta\right)\left(\tilde{u}_{\varepsilon}-\hat{u}_{\varepsilon}\right)=0 \quad \text { in } X, \\
& \left|\tilde{u}_{\varepsilon}-\hat{u}_{\varepsilon}+\varepsilon \frac{L}{\sigma} \frac{\partial}{\partial \nu}\left(\tilde{u}_{\varepsilon}-\hat{u}_{\varepsilon}\right)\right|_{\partial X} \leqslant \varepsilon^{2} C .
\end{aligned}
$$

We will use the ideas described above for the construction of correctors for the principal eigenvalue. Namely, from (55) we deduce that $z_{\varepsilon}$ is given by

$$
z_{\varepsilon}=\Phi-\varepsilon \frac{D}{\sigma} \cdot \nabla \Phi+\varepsilon w+O\left(\varepsilon^{2}\right)
$$

with $w$ given by

$$
(c-\sigma \gamma) w-k_{0} \Delta w=0,\left.\quad w\right|_{\partial X}=\left.\frac{1}{\sigma} L \frac{\partial \phi}{\partial \nu}\right|_{\partial X} .
$$


Therefore we deduce from $(59)^{5}$ the formula

$$
\begin{aligned}
\lim _{\varepsilon \rightarrow 0} \frac{1}{\varepsilon}\left(\omega_{\varepsilon}-\omega_{d}\right) & =\omega_{d} \lim _{\varepsilon \rightarrow 0}\left\langle-\varepsilon \frac{1}{\sigma} D \cdot \nabla \phi+\varepsilon w, \Psi_{\varepsilon}\right\rangle / \varepsilon \\
& =\omega_{d}\left\langle-\frac{D}{\sigma} \cdot \nabla \phi+w, \phi\right\rangle=\omega_{d}(w, \phi)_{L^{2}(X)}
\end{aligned}
$$

which finally gives the asymptotic expansion

$$
\omega_{\varepsilon}=\omega_{d}+\varepsilon \omega_{d}(w, \Phi)+O\left(\varepsilon^{2}\right) .
$$

In fact, to obtain an expansion of order 2 for the principal eigenvalue of $T_{\varepsilon}$, one can also directly use the operator $(c-\sigma \gamma)-k_{0} \Delta$ with the Robin boundary condition

$$
u+\varepsilon \frac{L}{\sigma} \frac{\partial u}{\partial \nu}=0
$$

Namely, we have

Proposition 2. Let $\mathscr{D}_{\varepsilon}$ be the dissipative operator $-(c-\sigma \gamma)+k_{0} \Delta$ defined in $X$ with the Robin boundary condition; then the principal eigenvalue $\tilde{\omega}_{\varepsilon}$ of $\mathscr{D}_{\varepsilon}$ converges when $\varepsilon$ goes to zero, to the principal eigenvalue $\omega_{d}$ of the operator $-(c-\sigma \gamma)+k_{0} \Delta$ with Dirichlet boundary condition. Furthermore, $\tilde{\omega}_{\varepsilon}$ admits an expansion of the form

$$
\tilde{\omega}_{\varepsilon}=\omega_{d}+\varepsilon a+O\left(\varepsilon^{2}\right)
$$

and the coefficient of $\varepsilon$ in (95) coincides with the coefficient of $\varepsilon$ in (93).

Proof. We introduce the principal eigenvalue and the principal eigenvector of $\mathscr{D}_{\varepsilon}$ :

$$
\begin{gathered}
-(c-\sigma \gamma) \Phi_{\varepsilon}+k_{0} \Delta \Phi_{\varepsilon}=\omega_{\varepsilon} \Phi_{\varepsilon} \quad(\text { in } X), \\
\Phi_{\varepsilon}+\varepsilon \frac{1}{\sigma} L \frac{\partial}{\partial \nu} \phi_{\varepsilon}=0 \quad(\text { on } \partial X) .
\end{gathered}
$$

We assume that the $L^{2}$ norm of $\Phi_{\varepsilon}$ is equal to one; $\tilde{\omega}_{\varepsilon}$ is negative and with the Raleigh-Ritz principle one can show that $\tilde{\omega}_{\varepsilon}$ is an increasing function of $\varepsilon$. It converges to a value $\tilde{\omega}$. On the other hand, $\phi_{\varepsilon}$ is positive (see, for instance, Amann [2]) and converges strongly in $L^{2}(X)$ to a positive function $\tilde{\Phi}$ which is the solution of the problem

$$
-(c-\sigma \gamma) \tilde{\Phi}+k_{0} \Delta \tilde{\Phi}=\tilde{\omega} \tilde{\Phi},\left.\quad \tilde{\Phi}\right|_{\partial X}=0 .
$$

This proves that $\tilde{\Phi}=\Phi$ and $\tilde{\omega}=\omega_{d}$. Next we introduce the solution $z_{\varepsilon}$ of the equation

$$
\mathscr{D}_{\varepsilon} \zeta_{\varepsilon}=\omega_{d} \Phi
$$

and we have (cf. (55)-(59))

$$
\omega_{d}\left\langle\Phi, \Phi_{\varepsilon}\right\rangle=\left\langle\mathscr{D}_{\varepsilon} z_{\varepsilon}, \Phi_{\varepsilon}\right\rangle=\left\langle z_{\varepsilon}, \mathscr{D}_{\varepsilon} \Phi_{\varepsilon}\right\rangle=\omega_{\varepsilon}\left\langle z_{\varepsilon}-\Phi, \Phi_{\varepsilon}\right\rangle+\omega_{\varepsilon}\left\langle\Phi, \Phi_{\varepsilon}\right\rangle
$$

or

$$
\frac{\omega_{d}-\omega_{\varepsilon}}{\varepsilon}\left\langle\phi, \phi_{\varepsilon}\right\rangle=\omega_{\varepsilon}\left\langle\frac{z_{\varepsilon}-\phi}{\varepsilon}, \phi_{\varepsilon}\right\rangle
$$

${ }^{5}$ All these results are due to Sentis and are described in [17] without application to the critical size. 
The proof follows the construction above in a fairly simpler fashion. ${ }^{6}$ We need only the expression up to order two of the solution of (98). We notice that $\left(z_{\varepsilon}-\phi\right) / \varepsilon$ is the solution of the elliptic problem

$$
\begin{gathered}
(c-\sigma \gamma) \frac{z_{\varepsilon}-\Phi}{\varepsilon}-k_{0} \Delta \frac{z_{\varepsilon}-\Phi}{\varepsilon}=0, \\
\frac{z_{\varepsilon}-\Phi}{\varepsilon}+\varepsilon \frac{1}{\sigma} L \frac{\partial}{\partial \nu} \frac{z_{\varepsilon}-\phi}{\varepsilon}=\frac{1}{\sigma} L \frac{\partial}{\partial \nu} \phi \quad \text { on } \partial X .
\end{gathered}
$$

Comparing with (84), it may easily be seen that $\left(z_{\varepsilon}-\phi\right) / \varepsilon \rightarrow w$. Then (100) yields $\left(\omega_{d}-\omega_{\varepsilon}\right) / \varepsilon \rightarrow \omega_{d}(w, \phi)_{L^{2}(X)}$. Q.E.D.

Coming back to the original principal eigenvalues $\Lambda_{\varepsilon}=+c+\omega_{\varepsilon}, \Lambda_{d}=+c+\omega_{d}$, $\Lambda_{\varepsilon}^{R}=c+\tilde{\omega}_{\varepsilon}$, we can summarize the above results in the following

THEOREM 6. Let $T_{\varepsilon}$ denote the transport operator

$$
T_{\varepsilon} u=-\frac{1}{\varepsilon} v \cdot \nabla u-\frac{\sigma}{\varepsilon^{2}}\left(u-\left(1+\varepsilon^{2} \gamma\right) K u\right)
$$

defined in $L^{2}(X \times V)$ with the absorbing boundary condition

$$
\left.u(x, v)\right|_{(\partial X \times V)_{-}}=0 .
$$

Let $\mathscr{D}$ denote the dissipative operator $k_{0} \Delta+\sigma \gamma$ defined in $L^{2}(X)$ with the Dirichlet boundary condition; finally, let $\mathscr{D}_{\varepsilon}$ be the elliptic operator $k_{0} \Delta+\sigma \gamma$, defined in $L^{2}(X)$ with the Robin, $\varepsilon$-dependent boundary condition

$$
u+\varepsilon \frac{1}{\sigma} L \frac{\partial u}{\partial \nu}=0 .
$$

We denote by $\Lambda_{\varepsilon}, \Lambda_{d}$ and $\Lambda_{\varepsilon}^{R}$ the corresponding principal eigenvalues of these three operators; then

(i) $\Lambda_{\varepsilon}$ converges to $\Lambda_{d}$ when $\varepsilon$ goes to zero,

(ii) $\Lambda_{\varepsilon}$ admits an expansion up to the order $\varepsilon$ of the form

$$
\Lambda_{\varepsilon}=\Lambda_{d}+\varepsilon \theta+O\left(\varepsilon^{2}\right),
$$

(iii) $\Lambda_{\varepsilon}^{R}$ admits, up to the order $\varepsilon$, the same expansion.

IV. Application to the computation of the critical size. As we have seen in the first section, the transport operator

$$
T u=-v \cdot \nabla u-\sigma(I-(1+\gamma) K) u,
$$

with absorbing boundary conditions, has its spectra in the region $\operatorname{Re} \lambda \leqslant \sigma \gamma$. Furthermore, in the region $-\sigma<\operatorname{Re} \lambda \leqslant \sigma \gamma$ the spectra is composed of eigenvalues of finite multiplicity. The number $\Lambda_{X}=\sup _{\lambda \in \Sigma(T)}\{\operatorname{Re} \lambda\}$ is the type of the semigroup, and for $\Lambda_{X}>-\sigma$ it is an eigenvalue of multiplicity one. In this section we will consider the transport operator in the open set $\eta X(\eta>0$ eventually large) and we will deduce from the results of the previous section the asymptotic behaviour of $\Lambda_{\eta_{X}}$ for $\eta$ going to infinity. $\Lambda_{X}$ is an increasing function of $X$ and we will use the diffusion approximation to define the critical size, i.e. the value of $\eta>0$ for which

${ }^{6}$ Some analogous ideas have been used in Kesavan [11] and Ciarlet and Kesavan [8]. 
one has $\Lambda_{\eta X}=0$. Due to the factor $\gamma$ it is more convenient to study the transport operator in the open set $(\eta /(1+\gamma)) X$. By a change of variable the eigenvalue equation is then written in the open set $X$ according to the formula

$$
-\frac{1}{\eta}(1+\gamma) v \cdot \nabla u-\sigma(I-(1+\gamma) K) u=\Lambda_{\eta} u,
$$

with the same absorbing boundary condition. (104) is also equivalent to the equation

$$
-\eta v \cdot \nabla u+\eta^{2} \sigma(I-K) u=\left(\frac{\Lambda_{\eta}-\sigma \gamma}{1+\gamma}\right) \eta^{2} u .
$$

We will denote by $\Lambda_{0}$ the principal eigenvalue of the operator $k_{0} \Delta$ defined in $X$ with the Dirichlet boundary condition and by $\Lambda_{\eta}^{R}$ the principal eigenvalue of the operator $k_{0} \Delta$, defined in $X$ with the Robin $\eta$-dependent boundary condition

$$
u+\frac{1}{\eta} \frac{L}{\sigma} \frac{\partial u}{\partial \nu}=0
$$

Now we can apply the results of Theorem 6 to equation (105) and obtain

THEOREM 7. When the size of the domain $\eta X$ goes to infinity, the principal eigenvalue $\Lambda_{\eta}$ converges to $\sigma \gamma$. Furthermore, the following asymptotic expansion is valid:

$$
\Lambda_{\eta}=\sigma \gamma+\frac{1}{\eta^{2}}(1+\gamma) \Lambda_{0}+\frac{1}{\eta^{3}} \theta+\frac{1}{\eta^{4}} O(1) .
$$

On the other hand, the terms of order 2 and 3 in $\eta$ in (107) are given by $\Lambda_{\eta}^{R}$ according to the formula

$$
\Lambda_{\eta}=\sigma \gamma+\frac{1+\gamma}{\eta^{2}} \Lambda_{\eta}^{R}+\frac{1}{\eta^{4}} O(1) .
$$

RemarK 4. One can show (cf. Appendix 1) that $\sigma \gamma$ is the type of the semigroup $\exp (t T)$ when $X=\mathbf{R}^{n}$. Therefore we have proved the continuity of this type for $X$ going to $\mathbf{R}^{n}$.

We already know that the equation which gives the $\eta$ corresponding to the critical size

$$
\sigma \gamma+\frac{1+\gamma}{\eta^{2}} \Lambda_{\eta}^{R}+\frac{1}{\eta^{4}} O(1)=0,
$$

has only one solution $\eta_{c}$. But $\Lambda_{\eta}^{R}$ is a negative number which goes to $\Lambda_{0}$ when $\eta$ goes to infinity. Therefore $z_{c}$ satisfies the relation

$$
\eta_{c}^{2}=-\frac{(1+\gamma) \Lambda_{\eta_{c}}^{R}}{2 \sigma \gamma}\left(1+\sqrt{1+4 \sigma \gamma O(1) /\left((1+\gamma) \Lambda_{\eta_{c}}^{R}\right)^{2}}\right) .
$$

The number $O(1)$ which appears in the right-hand side of (110) is bounded independently of $\gamma$. Therefore, for $\gamma$ small enough we have

$$
\sigma \gamma O(1) /\left((1+\gamma) \Lambda_{\eta_{c}}^{R}\right)^{2}<1 / 2
$$

and from (110) we deduce the bound

$$
\eta_{c}=\sqrt{-\Lambda_{\eta_{c}}^{R}(1+\gamma) / \sigma \gamma}(1+\gamma O(1)) .
$$


Formula (112) shows that $\eta_{c}$ is of the order of $\gamma^{-1 / 2}$ and therefore we finally have the two formulas

$$
\begin{aligned}
& \eta_{c}=\sqrt{-(1+\gamma) \Lambda_{0} / \sigma \gamma}(1+\sqrt{\gamma} O(1)), \\
& \eta_{c}=\sqrt{-(1+\gamma) \Lambda_{\eta_{c}}^{R} / \sigma \gamma}(1+\gamma(O(1)) .
\end{aligned}
$$

REMARK 5. It is interesting to compare the analysis done above and the justification of the diffusion approximation described by Larsen and Keller [13] or Blankenship and Papanicolaou [5]. In [5, 13] one starts with the equation

$$
\frac{\partial u_{\varepsilon}}{\partial t}+\frac{1}{\varepsilon} v \cdot \nabla u_{\varepsilon}+\frac{\sigma}{\varepsilon^{2}}\left(I-\left(1+\varepsilon^{2} \bar{\gamma}\right) K\right) u_{\varepsilon}=0
$$

and here we have considered the equation

$$
\frac{\partial u}{\partial t}+v \nabla u+\sigma(I-(1+\gamma) K) u=0
$$

in the open set $\eta X(1+\gamma)$. With $\varepsilon=\left(\eta_{0} /(1+\gamma)\right)^{-1}$ equation (116) turns out to be the equation

$$
\frac{\partial u_{\varepsilon}}{\partial t}+\varepsilon v \nabla u_{\varepsilon}+\sigma(I-(1+\gamma) K) u_{\varepsilon}=0
$$

and a change of scale in time; writing $t=\tau / \varepsilon^{2}$ reduces it to the equation

$$
\frac{\partial u_{\varepsilon}}{\partial \tau}+\frac{v}{\varepsilon} \nabla u_{\varepsilon}+\frac{\sigma}{\varepsilon^{2}}(I-(1+\gamma) K) u_{\varepsilon}=0 .
$$

Therefore we have shown that near the critical regime the mean free path is of the order of $\varepsilon$ and that the correct scale in time is given by $\tau=\varepsilon^{2} t$. Finally, if the open set $\eta X /(1+\gamma)$ is almost critical, (113) or (114) shows that we have $\eta \simeq 1 / \sqrt{\gamma}$ or $\varepsilon^{2} \simeq \gamma$ and this proves that near the critical regime the number $\gamma$, which characterises the cross section, is of the form $\gamma=\varepsilon^{2} \bar{\gamma}$, with $\bar{\gamma}$ of the order of the unity. This seems to be the only rigourous justification of the scaling done by [5 and 13] in the last term of (115).

In the §§II and III we have seen that in some sense the transport equation

$$
\frac{\partial u}{\partial t}+v \nabla u+\sigma(I-(1+\gamma) K) u=0
$$

is closely related to the diffusion equation

$$
\frac{\partial u}{\partial t}-k_{0} \Delta u-\sigma \gamma u=0
$$

with the Dirichlet boundary condition and that the approximation is better when the Robin boundary condition is used. For the critical size we will follow the same idea using (113) and (114). With the numbers $\Lambda_{0}$ and $\Lambda_{\eta}^{R}$ which appear in (107) and (108) it is easy to compute the critical size for the diffusion approximation. Namely, we have

Proposition 3. (i) Let $\Lambda_{0}$ denote the principal eigenvalue of the operator $k_{0} \Delta$ defined in $X$ with the Dirichlet boundary condition. Then the principal eigenvalue of the 
operator $k_{0} \Delta+\sigma \gamma$, defined in $\rho X$ with the Dirichlet boundary, is zero for $\rho$ given by the formula

$$
\rho_{c}=\sqrt{-\Lambda_{0} / \sigma \gamma} \text {. }
$$

(ii) Let $\Lambda^{R}$ denote the principal eigenvalue of the operator $k_{0} \Delta$, defined in $X$ with the $\rho$-dependent Robin boundary condition

$$
u+\frac{L}{\rho} \frac{1}{\sigma} \frac{\partial u}{\partial \nu}=0 \quad \text { on } \partial X
$$

then the principal eigenvalue of the operator $k_{0} \Delta+\sigma \gamma$, defined in $\rho X$ with the $\rho$-independent Robin boundary condition

$$
u+\frac{1}{\sigma} L \frac{\partial u}{\partial \nu}=0 \quad \text { on } \rho \partial X,
$$

is zero for $\rho_{C}^{R}$ given by the formula

$$
\rho_{C}^{R}=\sqrt{-\Lambda^{R} / \sigma \gamma} .
$$

The proof of this proposition is evident by scaling.

Finally, if we compare (113), (114) and Proposition 3 we have the final

THEOREM 8. Let $T$ denote the transport operator

$$
T u=-v \nabla u-\sigma(I-(1+\gamma) K) u
$$

in the open set $\rho X$. Let $k_{0} \Delta_{D}+\sigma \gamma$ denote the elliptic operator defined in the open set $\rho X$ with the Dirichlet boundary condition and with the constant $k_{0}$ computed according to the formulas of $\S \mathrm{II}$ in terms of the cross section operator $K$.

Finally, let $k_{0} \Delta_{D}+\sigma \gamma$ denote the same elliptic operator in the same open set $\rho X$ but with the Robin boundary condition

$$
u+\left.\frac{1}{\sigma} L \frac{\partial u}{\partial v}\right|_{\rho \partial X}=0 .
$$

Then for each of these three operators there exists a unique number $\rho_{c}^{T}, \rho_{c}^{D}, \rho_{c}^{R}$ for which the principal eigenvalue of the corresponding operator is zero in the open set $\rho X$. Furthermore, the difference between these three numbers goes to zero with $\gamma$ and we have

$$
\left|\rho_{c}^{T}(\gamma)-\rho_{c}^{D}\right|=\sqrt{\gamma} O(1) ; \quad\left|\rho_{c}^{T}(\gamma)-\rho_{c}^{R}\right|=\gamma O(1) ;
$$

the coefficient $O(1)$ appearing in the two equations being of the same order of magnitude.

V. Numerical examples in the case of the slab. As an illustration for (125) we consider the very classical example of the slab. The transport operator is then

$$
T u=-\mu \frac{\partial u}{\partial x}-u+\frac{1+\gamma}{2} \int_{-1}^{1} u\left(x, \mu^{\prime}\right) d \mu^{\prime},
$$

the open set $X$ is the interval ] $-1,1[$ and $\mu$ belongs to the set $V=[-1,1]$. Since we have $\int_{-1}^{1} \mu^{\prime} d \mu^{\prime}=0$, we obtain

$$
D(\mu)=\mu_{*}
$$


and the constant $k_{0}$ is given by

$$
k_{0}=\frac{1}{2} \int_{-1}^{1} \mu^{2} d \mu=\frac{1}{3} .
$$

On the other hand, in the present example the problem (73) is the classical problem

$$
\begin{gathered}
\mu \frac{\partial b}{\partial \eta}+b-\frac{1}{2} \int_{-1}^{1} b\left(x, \mu^{\prime}\right) d \mu^{\prime}=0 \text { for } \eta<0, \\
b(0, \mu)=\mu \text { for } \mu<0
\end{gathered}
$$

which has been studied extensively by Chandrasekhar [7]. In [7] it is proved that

$$
G(g)=\int_{0}^{1} \mu^{\prime} H\left(\mu^{\prime}\right) g\left(\mu^{\prime}\right) d \mu^{\prime}
$$

(where $H$ is the Chandrasekhar function). Then we have $L=L_{c}$ given by

$$
L_{c}=\int_{0}^{1} \mu^{\prime 2} H\left(\mu^{\prime}\right) d \mu^{\prime}
$$

and an approximate value of $L_{c}$ is $L_{c} \simeq 0.7104$.

Now, according to Amann [2] we know that the principal eigenvalue of the diffusion operator (with the Dirichlet or Robin boundary condition) is simple and that the corresponding eigenvector is positive. In the present example this eigenvector is an even function of $x$.

Therefore, the problem of the critical size for the Dirichlet and Robin boundary conditions turns out to be:

Find $\rho>0$ such that the equation

$$
\frac{1}{3} u^{\prime \prime}+\gamma u=0,
$$

with the boundary condition

$$
u(-\rho)=u(\rho)=0 \quad \text { (Dirichlet) }
$$

or

$$
u( \pm \rho) \pm L_{c} \frac{d u}{d \lambda}( \pm \rho)=0 \quad \text { (Robin) }
$$

has a nonzero positive solution. Since $u$ is even we deduce from (129) the expression $u(x)=\cos \sqrt{3 \gamma} x$ and from (130) and (131) the relations

$$
\begin{gathered}
\rho_{c}^{D}=\frac{\pi}{2}(3 \gamma)^{-1 / 2}, \\
\rho_{c}^{R}=(3 \gamma)^{-1 / 2} \tan ^{-1}\left((3 \gamma)^{-1 / 2} / L_{c}\right) .
\end{gathered}
$$

It is clear that for $\gamma$ very small these two formulas coincide; however, when $\gamma$ increases they differ with an error of the order of $\sqrt{\gamma}$, and the second gives more accurate results.

We can also write the operator $T$ defined by (103) in the following form:

$$
T u=-v \frac{\partial u}{\partial x}+\Sigma(K u-u)+\sigma \gamma u
$$

with $\Sigma=(1+\gamma) \sigma$. We can see that if $T$ is the operator

$$
T_{\varepsilon}=-\frac{1}{\varepsilon} v \nabla u_{\varepsilon}+\frac{\Sigma}{\varepsilon^{2}}\left(K u_{\varepsilon}-u_{\varepsilon}\right)+\sigma \gamma u \text {, }
$$


then the results of Theorem 4 are yet true with $\sigma$ replaced by $\Sigma$ in the Robin boundary condition and in the definition of $k_{0}$. Therefore, Theorems 5 and 6 remain true for the operator $T$ defined by (134) instead of (103) (with $\sigma$ replaced by $\Sigma$ in the Robin boundary condition and in the definition of $k_{0}$ ).

Then, if we come back to the operator $T$ which is of interest in this numerical example (where $\sigma=1$ ), we see that we may approximate the critical size of $T$ by the critical size $\rho_{0}^{D}$ (or $\rho_{0}^{R}$ ) of the diffusion operator

$$
\frac{1}{3(1+\gamma)} u^{\prime \prime}+\gamma u=0
$$

with the Dirichlet boundary condition

$$
u(-\rho)=u(\rho)=0
$$

or with the Robin boundary condition

$$
u( \pm \rho) \pm \frac{L_{c}}{1+\gamma} \frac{\partial u}{\partial x}( \pm \rho)=0
$$

That is to say that $\rho_{c}$ may also be approximated by

$$
\begin{gathered}
\rho_{0}^{D}=\frac{\pi}{2}(3 \gamma(1+\gamma))^{-1 / 2}, \\
\rho_{0}^{R}=(3 \gamma(1+\gamma))^{-1 / 2} \tan ^{-1}\left[\frac{1+\gamma}{[3 \gamma(1+\gamma)]^{1 / 2} L_{c}}\right] .
\end{gathered}
$$

Formulas (132), (133), (138) and (139) are equivalent for small $\gamma$. However when $\gamma$ increases, (132) and (138) differ from formulas (133) and (139) with an error of order $\gamma$ and the second ones are more accurate.

In the following table we compare the result of the various approximation formulas with some exact values (given by Case and Zweifel [6, p. 157, 207] and Williams [21, p. 210]).

\begin{tabular}{l|l|l|l|l|l}
$\gamma$ & $\rho_{c}^{D}$ & $\rho_{0}^{D}$ & $\rho_{c}^{R}$ & $\rho_{0}^{R}$ & $\rho$ exacts \\
\hline 0.01 & 8.07 & 9.02 & 8.36 & 8.32 & 8.32 \\
0.1 & 2.69 & 2.73 & 2.19 & 2.12 & 2.11 \\
0.277 & 1.72 & 1.52 & 1.09 & 1.02 & 1 \\
0.3 & 1.65 & 1.45 & 1.02 & 0.959 & 0.940 \\
0.6 & 1.17 & 0.926 & 0.60 & 0.545 & 0.515 \\
1 & 0.91 & 0.641 & 0.39 & 0.349 & 0.326 \\
1.807 & 0.67 & 0.403 & 0.23 & 0.203 & 0.1 \\
4.969 & 0.41 & 0.166 & 0.090 & 0.077 & 0.05 \\
13.26 & 0.25 & 0.066 & 0.035 & 0.029 & 0.01 \\
\hline
\end{tabular}

We see that the formula $\rho_{0}^{R}$ is the best.

When $\gamma$ increases from 0 to 1, it remains true up to 7\%. (It may be noticed that the formulas of approximation given by Case and Zweifel are valid only for $\gamma$ of order of 0.01 .) 
Appendix 1. Some remarks concerning the spectra of the transport operator in an unbounded domain. In the previous sections we have seen that the spectra of the operator

$$
T u=-v \nabla u-\sigma(I-(1+\gamma) K) u,
$$

defined in any $L^{p}(X \times V)(1 \leqslant p \leqslant \infty)$ with absorbing boundary conditions, was contained in the half-plane $\operatorname{Re} z<\sigma \gamma$ and that for $X$ going to $\mathbf{R}^{n}$, the type $\Lambda(X)$ of this semigroup converges to the number $\sigma \gamma$. This result is simply related to the behaviour of the transport operator in an unbounded domain and we have the following

THEOREM A.1. We assume that $X$ is an unbounded domain with $\mathbf{R}^{n} \backslash X$ contained in a ball of radius $\mathscr{S}_{0}$. We assume that the boundary $\partial X$ of $X$ is smooth and we denote by $T$ the transport operation

$$
T u=-v \cdot \nabla u-\sigma(u-(1+\gamma) K u)
$$

with $\sigma, \gamma$ two positive constants and $K$ the integral operator defined by (6) with the hypothesis (4) and (5), and with the usual absorbing boundary conditions. Then we have the following statements.

(i) For any $p(1 \leqslant p<\infty)$, $T$ is the generator of a semigroup in $L^{p}(X \times V)$.

(ii) $\forall p(1 \leqslant p<\infty)$, the spectra of $T$ in $L^{p}(X \times V)$ is contained in the half-plane $D=\{z \mid \operatorname{Re} z \leqslant \sigma \gamma\}$ and the point $\sigma \gamma$ belongs to the spectra of $T$.

(iii) If $X=\mathbf{R}^{n}, T$ is the generator of a group with spectra contained in the strip $D=\{z \mid-\sigma \leqslant \operatorname{Re} z \leqslant \sigma \gamma\}$ and the point $\sigma \gamma$ belongs to this spectra.

Proof. Except for the fact that $\sigma \gamma$ belongs to the spectra of $T$, all the results stated in this theorem can be derived by classical semigroup (or group) theory. Therefore we will only prove that $\sigma \gamma$ belongs to the spectra of $T$ in any $L^{p}(X \times V)$ space $(p<\infty)$. We consider the case $\mathbf{R}^{n} \backslash X \neq\{\varnothing\}$; the proof is the same when $X=\mathbf{R}^{n}$.

In fact, we have to show that 0 belongs to the spectra of the operator

$$
\tilde{T} T u=-v \cdot \nabla u-\sigma(1+\gamma)(u-K u) .
$$

For this purpose it is suficient to construct a sequence of functions $\varphi_{k}(x, v)$ with the following properties:

$$
\varphi_{k} \in D(\tilde{T}): \quad \lim _{k \rightarrow \infty}\left(\left\|\tilde{T} \varphi_{k}\right\|_{L^{p}(X \times V)} /\left\|\varphi_{k}\right\|_{L^{p}(X \times V)}\right)=0 .
$$

For the construction of these functions we introduce a family $\theta_{k}(s)$ of positive smooth functions defined in $\mathbf{R}_{+}$with the following properties:

$$
\begin{aligned}
0 \leqslant \theta_{k}(s) \leqslant 1, \quad\left|\theta_{k}^{\prime}(s)\right| \leqslant C<\infty \quad \forall s \in \mathbf{R}, \\
\theta_{k}(s)=0 \quad \text { for } s<\mathscr{S}_{0} \text { and } s>k+1, \\
\boldsymbol{\theta}_{k}(s)=1 \quad \text { for } \mathscr{S}_{0}+1<s<k .
\end{aligned}
$$


The functions $\varphi_{k}(x)=\theta_{k}(|x|)$ belong to the domain of $\tilde{T}$, they are independent of $v$ and they satisfy the relation

$$
\tilde{T} \varphi_{k}=v \cdot \frac{x}{|x|} \theta_{s}^{\prime}(x)
$$

Therefore (A.1) is easy to prove.

Appendix 2. The conservative Milne problem in the half-space. As we have seen, the solution of the conservative Milne problem (77)-(79) plays a crucial role in the construction of the boundary layer corrector. And it is the only rigourous way to introduce the extrapolation length. This problem has been considered by many authors in several explicit cases (cf. Chandrasekhar [5]). However, the general case has only been treated by Bensoussan, Lions and Papanicolaou [4]. Their method relies on probability theory. This turns out to be very close to intuition and provides efficient tools for the proof. On the other hand, these proofs are rather long and seem to have no relation with the classical spectral theory. Therefore in this appendix we show that the problem (77)-(79) can be handled by classical analysis. Starting from (77)-(79) and noticing that $\tilde{x}$ and $\nu(\tilde{x})$ are parameters, we introduce the variables $v_{n}, \hat{v}$ defined by

$$
v_{n}=v \cdot \nu(\hat{x}), \quad \hat{v}=v-(v \cdot \nu(\hat{x})) \nu(\hat{x})
$$

and changing $\eta$ in $-\eta / \sigma$ we consider the following problem in $\mathbf{R}_{+} \times V$ :

$$
\begin{gathered}
v_{n} \frac{\partial u}{\partial \eta}+(I-K) u=0 \text { in } \mathbf{R}_{+} \times V, \\
u(0, v)=g(v) \text { for } v \in V_{+}=\left\{v \mid v_{n}>0\right\} .
\end{gathered}
$$

The spectra of the transport operator $T_{u} \equiv-v_{n}(\partial u / \partial \eta)-u+K u$ with absorbing boundary condition is contained in the half-space $\operatorname{Re} z<0$ (in any $L^{p}\left(\mathbf{R}_{+} \times V\right.$ ) space $1 \leqslant p \leqslant \infty)$. Therefore for any $\varepsilon>0$ there exists a unique solution of the problem

$$
\begin{gathered}
v_{n} \frac{\partial u_{\varepsilon}}{\partial \eta}+u_{\varepsilon}-K u_{\varepsilon}+\varepsilon u_{\varepsilon}=h ; \quad\left(h \in L^{p}\left(\mathbf{R}_{+} \times V\right)\right), \\
u_{\varepsilon}(0, v)=0 \quad \text { for } v \in V_{+} .
\end{gathered}
$$

By a superposition argument one can deduce that for any $\varepsilon>0$ and any $g \in L^{p}\left(V_{+}\right)$ there exists a unique solution of

$$
\begin{gathered}
v_{n} \frac{\partial u_{\varepsilon}}{\partial \eta}+u_{\varepsilon}-K u_{\varepsilon}+\varepsilon u_{\varepsilon}=0 \quad \text { in } \mathbf{R}_{+} \times V, \\
u_{\varepsilon}(0, v)=g(v) \text { for } v \in V_{+} .
\end{gathered}
$$

On the other hand, Theorem A.1 applies to the present case and this implies that 0 belongs to the spectra of $T$ in any $L^{p}\left(\mathbf{R}_{+} \times V\right)$ space $(1 \leqslant p<\infty)$. Therefore, in general there is no function $u \in L^{p}\left(\mathbf{R}_{+} \times V\right)$ which satisfies (A.6) and (A.7). This is related to the following fact which will appear below. A bounded solution of (A.6), (A.7), in general, does not go to zero for $\eta$ going to infinity, but to a nonzero constant $H(g)$, as it will be proved below. Therefore we will proceed as follows: 
first, we prove the existence of a bounded soltuion of (A.6), (A.7) and show that this solution is positive when $g$ is positive. Second, we describe the asymptotic behaviour for $x \rightarrow \infty$ of any bounded solution of (A.6), (A.7). Finally, we show the uniqueness of the bounded solution and therefore we define a linear continuous map $H$ : $L^{\infty}\left(V_{+}\right) \rightarrow \mathbf{R}$ by the formula

$$
H(g)=\lim _{x \rightarrow \infty} u(x, v) .
$$

Theorem A.2. For any $g \in L^{\infty}\left(V_{+}\right)$there exists at least one bounded solution of the problem

$$
\begin{gathered}
v_{n} \frac{\partial u}{\partial \eta}+u-\int_{V} f\left(v, v^{\prime}\right) u(\eta, v) d v^{\prime}=u \quad \text { in } \mathbf{R}_{+} \times V, \\
u(0, v)=g(v), \quad v \in V_{+} .
\end{gathered}
$$

This solution satisfies the maximum principle

$$
|u(x, v)| \leqslant \sup _{v \in V_{+}}|g(v)|
$$

and is nonnegative whenever $g$ is positive.

Proof. We know that the problem (A.10), (A.11) has a unique solution $u_{\varepsilon}$, and that this solution is nonnegative when $g$ is nonnegative. For this solution $u_{\varepsilon}$, one can apply the maximum principle and prove the estimate

$$
\left|u_{\varepsilon}(x, v)\right| \leqslant \sup _{v \in V_{+}}|g(v)|, \quad(x, v) \in \mathbf{R}_{+} \times V .
$$

Since (A.13) is uniform with respect to $\varepsilon$, one can extract from the family $u_{\varepsilon}$ a subfamily $u_{\varepsilon^{\prime}}$ which converges in $L^{\infty}\left(\mathbf{R}_{+} \times V\right)$ weak* to a function $u$. By a classical limiting argument and using some trace theorems (cf. Bardos [2]), one concludes that this function satisfies both (A.6) and (A.7).

For the next step (the asymptotic behaviour of any bounded solution) it will be convenient to have at our disposal the following

Lemma A.1. Let $u \in L^{\infty}\left(\mathbf{R}_{+} \times V\right)$ be a solution of the equation

$$
v_{n} \frac{\partial u}{\partial \eta}+(I-K) u=0 \quad \text { in } \mathbf{R}_{+} \times V .
$$

Then $\partial u / \partial \eta$ is also a solution of the equation (A.14), which is uniformly bounded in $\left.L^{\infty}(] 2, \infty[\times V)\right)^{7}$

Proof. In the domain $] 0,6[\times V=X \times V$ we consider the transport operator

$$
T u=v_{n} \nabla u-(I-K) u
$$

with the boundary conditions

$$
u(0, v)=0 \text { for } v_{n}>0, \quad u(6, v)=0 \text { for } v_{n}<0
$$

zero does not belong to the spectra of this operator in any $L^{p}(X \times V)$ space, including $L^{\infty}(X \times V)$.

\footnotetext{
${ }^{7}$ Instead of 2 one can choose any strictly positive number.
} 
Now let $u \in L^{\infty}\left(\mathbf{R}_{+} \times V\right)$ be the solution of (A.10), (A.11) and let

$$
\left\{\begin{array}{l}
\varphi \text { be a smooth positive function of } \eta \text { which satisfies the relation: } \\
\varphi(\eta)=0 \text { for } 0 \leqslant \eta \leqslant 1 \text { and } \eta \leqslant 5 \\
\varphi(\eta)=1 \text { for } 2 \leqslant \eta \leqslant 4
\end{array}\right.
$$

Then the function $h(\eta, v)=d(\varphi(\eta) u) / d \eta$ is zero on $\partial X$ it coincides with $\partial u / \partial \eta$ for $2 \leqslant \eta \leqslant 4$ and it satisfies the equation

$$
\begin{aligned}
& v_{n} \frac{\partial}{\partial \eta}\left(\frac{\partial}{\partial \eta}(\varphi(\eta) u)\right)+(I-K) \frac{\partial}{\partial \eta}(\varphi(\eta) u) \\
& \quad= \frac{\partial}{\partial \eta}\left[v_{n} \frac{\partial}{\partial \eta}(\varphi(\eta) u)+(I-K)(\varphi(\eta) u)\right] \\
& \quad= \frac{\partial}{\partial \eta}\left[\varphi(\eta)\left(v_{n} \frac{\partial u}{\partial \eta}+(I-K) u\right)+v_{n} \varphi^{\prime}(\eta) u\right] \\
& \quad=\varphi^{\prime}(\eta) v_{n} \frac{\partial u}{\partial \eta}+v_{n} \varphi^{\prime \prime} \\
& \quad=-\varphi^{\prime}(\eta)(I-K) u+v_{n} \varphi^{\prime \prime}(\eta) u
\end{aligned}
$$

Therefore $h(\eta, v)$ is the solution of the transport equation

$$
v_{n} \frac{\partial h}{\partial \eta}+(I-K) h=L(\eta, v)
$$

with the boundary condition (A.16); by the maximum principle we have

$$
|h(\eta, v)|_{L^{\infty}(X \times V)} \leqslant C_{0}|L(\eta, v)|_{L^{\infty}(X \times V)} \leqslant C_{0} C_{1}|u|_{L^{\infty}(X \times V)} .
$$

This proves the estimate

$$
\sup _{\substack{2 \leqslant \eta \leqslant 4 \\ v \in V}} \frac{\partial u}{\partial \eta}(\eta, v) \leqslant C_{0} C_{1}|u|_{L^{\infty}(X \times v)}
$$

Finally, one observes that the method of the proof and, therefore, the constants $C_{0}$ and $C_{1}$ are translation invariant and this completes the proof of Lemma A.1.

For any function $u(x, v)$ we introduce the density function $q_{u}=\int_{V} u(x, v) d v$ and the natural decomposition

$$
u(x, v)=q_{u}(x)+w_{u}(x, v) \text { with } \int_{V} w_{u}(X, v) d v=0 .
$$

Then we have

TheOREM A.3. Let $u \in L^{\infty}\left(\mathbf{R}_{+} \times V\right)$ be a solution of the conservative Milne problem

$$
v_{n} \frac{\partial u}{\partial \eta}+(I-K) u=0 \quad \text { in } \mathbf{R}_{+} \times V
$$

let

$$
q_{u}=\int_{V} u(\eta, v) d v \quad \text { and } \quad w_{u}(\eta, v)=u(\eta, v)-q_{u}(\eta)
$$


denote the natural decomposition of $u$. Then there exist a strictly positive number $\alpha$ such that the estimate

$$
\left(\int_{V}|w(\eta, v)|^{2} d v\right)^{1 / 2}+\left|\frac{\partial q}{\partial \eta}(\eta)\right| \leqslant C_{u} C^{-\alpha \eta}
$$

is true, where the constant $C$ depends on $u$ (but not on $\eta$ ). Futhermore, for any $u \in L^{\infty}\left(\mathbf{R}_{+} \times V\right)$, which is a solution of the conservative Milne problem, $q_{n}(\eta)$ converges exponentially to a constant $H(u)$, i.e. we have the estimate

$$
\left|q_{u}(\eta)-H(u)\right| \leqslant C_{u} e^{-\alpha \eta} .
$$

Proof. In the proof we omit the index $u$ for $q_{u}$ and $w_{u}$. $K$ is a compact selfadjoint positive operator with spectra in the interval $[0,1]$ and 1 is a simple eigenvalue. Therefore, there exists a constant $\alpha_{0}>0$, such that for any $w \in \operatorname{Ker}(I-K)^{\perp}$ (that is to say, $(w, 1)=0)$, we have

$$
((I-K) w, w) \geqslant \alpha_{0}\|w\|^{2} \cdot 8
$$

Multiplying (A.22) by $u$, we obtain, after integration over [0, $x \times V]$, the relation

$$
\alpha_{0} \int_{0}^{x}\|w(\eta)\|^{2} d \eta+\left(v_{n}, u^{2}(x)\right) \leqslant\left(v_{n}, u^{2}(0)\right) .
$$

Then the function $\eta \rightarrow|w(\eta)|$ is in $L^{2}(0,+\infty)$ and therefore there exists a sequence $\eta_{p}$ converging to $\infty$ such that

$$
\lim _{p \rightarrow \infty}\left\|w\left(\eta_{p}\right)\right\|^{2}=0
$$

and thus

$$
\lim _{p \rightarrow \infty}\left(v_{n}, w\left(\eta_{p}\right)\right)=0
$$

On the other hand, if we integrate (A.22) over $V$, we obtain

$$
0=\frac{\partial}{\partial \eta} \int_{V} v_{n}(q+w(\eta)) d v+\int_{V}(u-K U) d v=\frac{\partial}{\partial \eta}\left(v_{n}, w(\eta)\right)
$$

Then $\left(v_{n}, w(\eta)\right)$ does not depend on $\eta$, and due to (A.28) we have, for any $\eta>0$,

$$
\left(v_{n}, w(\eta)\right)=0 \text {. }
$$

Therefore we obtain

$$
\left(v_{n}, u^{2}\right)=\left(v_{n}, w^{2}\right)+2\left(v_{n}, w\right)=\left(v_{n}, w^{2}\right) .
$$

But multiplying (A.22) by $u$ and integrating over $[x, y] \times V$ we obtain

$$
\left(v_{n}, w(y)\right)^{2}-\left(v_{n}, w(x)\right)^{2}=-\int_{x}^{y}((I-K) u, u) d \eta \leqslant 0 .
$$

Thus the function $\eta \rightarrow\left(v_{n}, w(\eta)^{2}\right)$ is decreasing; but due to (A.27) we see that $\left(v_{n}, w(\eta)^{2}\right)$ goes to zero with $\eta$ going to infinity. Therefore, for any positive $x$ we have

$$
\left(v_{n}, u^{2}(x)\right)=\left(v_{n}, w^{2}(x)\right) \geqslant 0 .
$$

\footnotetext{
${ }^{8}(\cdot, \cdot)$ denotes the scalar product in $L^{2}(V)$ and \|\| the norm in $L^{2}(V)$.
} 
Finally, we multiply (A.22) by $u e^{2 \alpha \eta}$ and using (A.25) we obtain, after integration over $V$,

$$
\text { (A.33) } \begin{aligned}
& 0 \geqslant \frac{1}{2} \frac{\partial}{\partial \eta}\left[\left(v_{n}, u^{2}(\eta)\right) e^{2 \alpha \eta}\right]+\alpha_{0}\|w(\eta)\|^{2} e^{2 \alpha \eta}-\alpha\left(v_{n}, u^{2}(\eta)\right) e^{2 \alpha \eta} \\
& \geqslant \frac{1}{2} \frac{\partial}{\partial \eta}\left[\left(v_{n}, u^{2}(\eta)\right) e^{2 \alpha \eta}\right]+\left(\alpha_{0}-\alpha_{1} \alpha\right)\|w(\eta)\|^{2} e^{2 \alpha \eta} .
\end{aligned}
$$

We integrate (A.33) over the inverval $[0, x]$ and using the relation (A.32) we have the estimate

$$
\text { (A.34) } e^{2 \alpha x} \int_{V} v_{n}|w(x, v)|^{2} d v+\left(\alpha_{0}-\alpha_{1} \alpha\right) \int_{0}^{x} \int_{V} e^{2 \alpha \eta}|w(\eta, v)|^{2} d v d \eta \leqslant C_{u} \text {. }
$$

We conclude that with $\alpha<\alpha_{0} / \alpha_{1}$ we have

$$
0 \leqslant\left(v_{n}, w(x)^{2}\right) \leqslant C_{u} e^{-2 \alpha x}
$$

and

$$
\int_{0}^{\infty} e^{2 \alpha \eta}\|w(\eta)\|^{2} d \eta \leqslant C_{u} .
$$

Now we use Lemma A.1. The function $\partial u / \partial \eta$ belongs to $L^{\infty}(] 2, \infty[\times V)$ and is a solution of the equation

$$
\eta \frac{\partial}{\partial \eta}\left(\frac{\partial u}{\partial \eta}\right)+(I-K)\left(\frac{\partial u}{\partial \eta}\right)=0
$$

Therefore we introduce the decomposition

$$
\frac{\partial u}{\partial \eta}=\frac{d q}{d \eta}+\frac{\partial w}{\partial \eta}(\eta, v)
$$

The estimates described above for $w$ apply with no modification to $\partial w / \partial \eta$ and since $\partial u / \partial \eta$ is bounded in $L^{\infty}(] 2, \infty[\times V)$ by $\|u\|_{L^{\infty}\left(\mathbf{R}_{+} \times V\right)}$ we obtain the estimate

$$
\int_{\eta}^{\infty} e^{2 \alpha \eta}\left\|\frac{\partial w}{\partial \eta}(\eta)\right\|^{2} d \eta \leqslant C_{u}^{\prime}
$$

From (A.39) and (A.36) we deduce by an elementary interpolation (by use of the Cauchy-Schwarz inequality) the relation

$$
\|w(\eta)\| \leqslant C_{u} e^{-\alpha \eta} .
$$

Now we multiply (A.22) by $v$ and we integrate over $V_{+}=\left\{v \in V \mid v_{n}>0\right\}$; we obtain

(A.41) $\left(\int_{V_{+}} v_{n}^{2} d v\right) \frac{\partial q}{\partial \eta}+\frac{\partial}{\partial \eta} \int_{V_{+}} v^{2} w(\eta, v) d v=\int_{V_{+}}(K-I) w(\eta, v) v d v$.

But we have, by the Cauchy-Schwarz inequality,

$$
\begin{gathered}
\left|\int_{V_{+}} v^{2} w(\eta, v) d v\right| \leqslant C_{0} \int_{V_{+}} w(\eta, v)^{2} d v \leqslant C_{1}|| w(\eta) \|^{2}, \\
\left|\int_{V_{+}}(K-I) w(\eta, v) v d v\right| \leqslant C_{2}\|w(\eta)\|^{2} .
\end{gathered}
$$


Then, due to (A.40) the relation (A.41) yields the estimate

$$
\left|\frac{\partial q}{\partial \eta}\right| \leqslant D_{u}\left(C_{1}+C_{2}\right) e^{-\alpha \eta}
$$

With (A.40) and (A.42) we have proved the relation (A.23). Finally, we deduce from (A.42) that $q(\eta)$ converges, for $\eta$ going to infinity, to a constant $H(u)$ and that the estimate (A.24) is valid.

Now we can show the uniqueness of the solution of the Milne problem.

THEOREM A.4. Let $u_{i}(x, v) \in L^{\infty}\left(\mathbf{R}_{+} \times V\right)(i=1,2)$ be two solutions of the conservative Milne problem

$$
v_{n} \frac{\partial u_{i}}{\partial \eta}+(I-K) u_{i}=0 \quad \text { in } \mathbf{R}_{+} \times V
$$

Then if $u_{1}(0, v)$ and $u_{2}(0, v)$ coincide for $v_{n}>0$, they coincide everywhere.

Proof. ${ }^{9}$ We already know that $u_{1}$ and $u_{2}$ converge exponentially to some constant $c_{1}$ and $c_{2}$ for $x$ tending to infinity. Now we introduce the function

$$
w=\left(u_{1}-c_{1}\right)-\left(u_{2}-c_{2}\right)
$$

$w$ converges to zero exponentially when $x$ goes to infinity and for $x=0$ we have

$$
w(0, v)=c_{2}-c_{1}=\delta \text { for } v_{n}>0 .
$$

Furthermore, $w(x, v)$ is a solution of the transport equation

$$
v_{n} \frac{\partial w}{\partial \eta}+(I-K) w=0 .
$$

Multiplication of (A.44) by $w$ and integration over $\mathbf{R}_{+} \times V$ gives the relation

$$
\int_{0}^{\infty} \int_{V}((I-K) w \cdot w) d v d \eta=\int_{V} v_{n}(w(0, v))^{2} d v+\delta \int_{V_{+}} v_{n} d v
$$

Therefore we have

(A.46)

$$
\delta \int_{V_{+}} v_{n} d v=\int_{V_{-}}-v_{n} w(0, v) d v \leqslant\left(\int_{V_{-}}-v_{n} d v\right)^{1 / 2}\left(\int_{V_{-}}-v_{n}|w(0, v)|^{2} d v\right)^{1 / 2}
$$

by the Cauchy-Schwarz relation ${ }^{10}$ since we have, by symmetry,

$$
\int_{V_{-}} v_{n} d v=\int_{V_{-}}-v_{n} d v=\gamma
$$

We deduce from (A.38) the relation

$$
\delta^{2} \int_{V_{+}} v_{n} d v \leqslant \int_{V_{-}}-v_{n}(w(0, v))^{2} d v
$$

From (A.48) we deduce that the right-hand side of (A.45) is nonpositive. This implies that we have $((I-K) w, w)=0$. Therefore $w(, v)$ is independent of $v$ and

\footnotetext{
${ }^{9}$ The proof has been suggested to us by J. L. Lions.

${ }^{10} V_{ \pm}=\left\{v \in V \mid v_{n} \gtrless 0\right\} ;-v_{n}$ is positive on $V_{-}$.
} 
$(I-K) w \equiv 0$. This implies that $\partial w(\eta) / \partial \eta=0$ and $w$ itself is identically equal to zero. This completes the proof of Theorem A.4.

With Theorems A.2, A.3 and A.4 we have proved that for any $g \in L^{\infty}\left(V_{-}\right)$there exists a unique function $u \in L^{\infty}\left(\mathbf{R}_{+} \times V\right)$ which is the solution of the problem

$$
\begin{gathered}
v_{n} \frac{\partial u}{\partial \eta}+(I-K) u=0 \text { in } \mathbf{R}_{+} \times V, \\
u(0, v)=g(v) \text { for } v \in V .
\end{gathered}
$$

This function converges exponentially fast to a constant $H_{g}$ for $\eta$ going to $-\infty$. Furthermore, if $g$ is a nonnegative function, $u$ and $H_{g}$ are also nonnegative. Now if we change $\eta$ into $-\eta, \mathbf{R}_{+} \times V$ into $\mathbf{R}_{-} \times V$, and $V_{+}$into $V_{-}$, we obtain statements (i), (ii), (iii) and (iv) of Theorem 4. The proof of statement (v) is given in the following

Proposition A.1. Let $v_{n}$ be the component of $v \in V$ in the direction $\eta$ in the space $\mathbf{R}_{-} \times V$, let $D_{n}(v)$ be the solution of the equations

$$
(I-K) D_{n}(v)=v_{n}, \quad \int_{V} D_{n}(v) d v=0,
$$

and let $u(\eta, v)$ be the solution of the conservative Milne problem

$$
\begin{gathered}
v_{n} \frac{\partial u}{\partial \eta}+(I-K) u=0 \quad \text { in } \mathbf{R}_{-} \times V, \\
\left.u(0, v)\right|_{V_{+}}=\left.D_{n}(v)\right|_{V_{+}} .
\end{gathered}
$$

Then the number $H\left(D_{n}\right)=\lim _{\eta \rightarrow \infty} u(\eta, v)=-L$ is negative.

Proof. If we multiply the first equation of (A.43) by $D_{n}(v)$ and integrate over $\mathbf{R}_{-} \times V$, we obtain

$$
\begin{aligned}
-\left(v_{n} L, D_{n}\right)+ & \left(v_{n} u(0, v), D_{n}\right)+\int_{-\infty}^{0}\left((I-K) D_{n}(v), u(\eta)\right) d \eta \\
& =\int_{-\infty}^{0}\left(v_{n}, q_{u}(\eta)\right) d \eta=\int_{-\infty}^{0}\left(v_{n}, w_{u}(\eta)\right) d \eta=0
\end{aligned}
$$

(cf. (A.28)). From (A.52) and from the relation

$$
\int_{V} v_{n} D_{n}(v) d v=e_{0}>0
$$

(cf. (45)) we deduce the formula

$$
L=-\frac{1}{e_{0}}\left(v_{n} u(0, v), D_{n}(v)\right)
$$

Now we have

$$
\text { (A.54) } \begin{aligned}
\left(v_{n} u(0, v), D_{n}(v)\right) & =\int_{V_{-}} v_{n}\left(D_{n}(v)\right)^{2} d v+\int_{V_{+}} v_{n} D_{n}(v) u(0, v) d v \\
& =\int_{V_{-}}-\left|v_{n}\right|\left(D_{n}(v)\right)^{2} d v+\int_{V_{+}} v_{n} D_{n}(v) u(0, v) d v
\end{aligned}
$$


With the symmetries of $V, v_{n}$ and $D_{n}(v)$, and with the Cauchy-Schwarz inequality we obtain

(A.55)

$$
\begin{aligned}
& \left(v_{n} u(0, v), D_{n}(v)\right) \\
& \quad \leqslant-\int_{V_{-}}\left|v_{n}\right|\left(D_{n}(v)\right)^{2} d v+\left(\int_{V_{+}} v_{n}\left(D_{n}(v)\right)^{2} d v\right)^{1 / 2}\left(\int_{V_{+}} v_{n}\left(u(0, v)^{2} d v\right)^{1 / 2}\right. \\
& \quad \leqslant\left(\int_{V_{+}} v_{n}\left(D_{n}(v)\right)^{2} d v\right)^{1 / 2}\left(\int_{V_{-}} v_{n}\left(D_{n}(v)\right)^{2} d v+\left(\int_{V_{+}} v_{n}(u(0, v))^{2} d v\right)^{1 / 2}\right) \\
& \quad \leqslant\left(\int_{V_{+}} v,\left(D_{n}(v)\right)^{2} d v\right)^{1 / 2}\left(\int_{V} v_{n}|u(0, v)|^{2} d v\right)^{1 / 2} .
\end{aligned}
$$

Finally we multiply the first equation of (A.51) by $u(\eta)$ and obtain

$$
\frac{d}{d \eta}\left(v,(u(\eta))^{2}\right)=-((I-K) u(\eta), u(\eta)) \leqslant 0 .
$$

We know that $\left(v,(u(\eta))^{2}\right)$ goes to zero when $\eta$ goes to $-\infty$. Therefore we deduce from (A.56) that the last term of (A.55) is negative, and the proof of Proposition A.1 is complete.

REMARK A.1. The results of Appendix 2 apply also with minor changes to the Milne problem

$$
\begin{gathered}
v_{n} \frac{\partial u}{\partial \eta}+\sigma(\eta)\left(u-\int_{V} f\left(\eta, v, v^{\prime}\right) u\left(x, v^{\prime}\right) d v^{\prime}\right)=0 \text { in } \mathbf{R}_{-} \times V \\
u(0, v)=g(v) \text { for } v \in V_{+}
\end{gathered}
$$

with the hypothesis $0<\sigma_{e} \leqslant \sigma(\eta) \leqslant \sigma_{u}<\infty ; 0<\pi_{l} \leqslant f\left(\eta, v, v^{\prime}\right) \leqslant \pi_{u}<\infty$, $f\left(\eta, v, v^{\prime}\right)=f\left(\eta, v^{\prime}, v\right) ; \int_{V} f\left(\eta, v, v^{\prime}\right) d v^{\prime}=1$, where $\sigma_{e}, \sigma_{u}, \pi_{l}$ and $\pi_{u}$ are constant.

First, one can suppress the term $\sigma(\eta)$ by the change of variable defined according to the formula

$$
\tilde{\eta}=\int_{0}^{\eta} \frac{d s}{\sigma(s)}
$$

Second, one can see that the proof of Theorems A.2 and A.4 can be done with no modification. The same remarks apply to the proof of Theorem A.3 up to the estimate (A.40). The only thing which has to be improved, with some convenient hypothesis on the dependence of $f\left(\eta, v, v^{\prime}\right)$ with respect to $\eta$, is Lemma A.1 and this is left to the reader.

REMARK A.2. It is also possible to generalize some of the results of Appendix 2 to a conservative Milne problem in more than one space variable. Namely, we denote 
by $X$ the exterior of a bounded obstacle with smooth boundary $\partial X$ and we consider the Milne conservative problem

$$
\begin{gathered}
v \cdot \nabla u+\sigma(x)\left(u-\int_{V} f\left(x, v, v^{\prime}\right) u\left(x, v^{\prime}\right) d v^{\prime}\right)=0 \quad \text { in } X \times V, \\
u(x, v)=g(x, v) \text { for }(x, v) \in(\partial X \times V)_{-}
\end{gathered}
$$

with $\sigma$ and $f$ satisfying the usual hypothesis and $g$ belonging to $L^{\infty}(\partial X \times V)$. It is easy to follow the proof of Theorem A.2 and to obtain the existence of a bounded solution. On the other hand, if one assumes that we have

$$
\lim _{R \rightarrow \infty} \int_{|X|=R} d \sigma_{R} \int_{V} v \cdot \frac{x}{|X|} w(x, v) d v=0
$$

(with the usual definition of $w$ ) for any bounded solution, then one can also adapt the proof of Theorem A.4 and obtain the uniqueness of the solution in the class of bounded functions. However, we have not been able to prove (A.62) for any bounded solution in more than one space variable, due to the appearance of a factor proportional to the surface of the ball of radius $R$ in $\mathbf{R}^{n}$; therefore, we are not able to prove the analoguous version of Theorem A.3 in more than one space variable and the uniqueness of the bounded solution without supplementary hypotheses on the asymptotic behaviour of this solution. On the other hand, we ignore if the Milne conservative problem in more than one space variable has any physical significance or any application.

\section{REFERENCES}

1. G. Albertoni and B. Montagnin, On the spectrum of neutron transport equations in finite bodies, J. Math. Anal. Appl. 13 (1966), 19-48.

2. H. Amann, Nonlinear operator in ordered Banach spaces and some applications to nonlinear boundary value problems in nonlinear operator and the calculus of variations (Proc., Bruxelles, 1975), Lecture Notes in Math., vol. 543, Springer-Verlag, Berlin, 1976, pp. 1-55.

3. C. Bardos, Problèmes aux limites pour les équations aux dérivées partielles du premier order, Ann. Sci. Ecole Norm. Sup. 4 (1969), 185-233.

4. A. Bensoussan, J. L. Lions, and G. C. Papanicolaou, Boundary lavers and homogenization of transport processes, J. Publ. RIMS Kyoto Univ. 15 (1979), 53-157.

5. G. Blankenship and G. C. Papanicolaou, Stability and control of stochastic systems with wide-band noise disturbance. I, SIAM J. Appl. Math. 34 (1978), 437-476.

6. K. M. Case and P. F. Zweifel, Linear transport theory, Addison-Wesley, Reading, Mass.

7. S. Chandrasekhar, Radiative transfer, Dover, New York, 1950.

8. P. G. Ciarlet and S. Kesavan, Two dimensional approximation of three dimensional eigenvalue problems in plate theory, Comput. Methods Appl. Mech. Engrg. 26 (1981), 145-172.

9. F. Harris, The theory of branching process, Springer-Verlag, Berlin, 1963.

10. T. Kato, Perturbation theory for linear operators, Springer-Verlag, Berlin and New York, 1976.

11. S. Kesavan, Homogenisation of eigenvalue problems, Appl. Math. Optim. 5 (1979), 153-168.

12. M. G. Krein and M. A. Rutman, Linear operators leaving invariant a cone of positive functions in a Banach space, Trans. Amer. Math. Soc. 26 (1950).

13. E. Larsen and J. B. Keller, Asymptotic solutions of neutron transport problems, J. Math. Phys. 15 (1974), 75-81.

14. J. Lehner and G. Wing, On the spectra of an asymmetric operator arising in the transport theory of neutrons, Comm. Pure Appl. Math. 8 (1955), 217-234.

15. M. Mokhtar Kharroubi, Propriétes spectrales de l'opérateur de transport dans les cas anisotrope, Thèse 3ème cycle, Université de Paris VI, 1982. 
16. R. Sentis, Analyse asymptotique d'équation de transport, Thèse, Université de Paris-Dauphine, Décembre 1981.

17. , Study of the corrector of the eigenvalue of a transport operator, SIAM J. Math. Anal. 15 (1984).

18. Y. Shmulyan, Completely continuous perturbation of operator, Dokl. Akad. Nauk SSSR 101 (1955), 33-38.

19. I. Vidav, Existence and uniqueness of nonnegative eigenfunctions of the Boltzmann operator, J. Math. Anal. Appl. 22 (1968), 144-155.

20. __ Spectrum of perturbed semi-groups with application to transport theory, J. Math. Anal. Appl. 30 (1970), 244-279.

21. M. Williams, Mathematical methods in particle transport theory, Butterworth, London, 1971.

Centre de Mathématiques Appliquées, École Normale Supérieure, 75005 Paris, France

Départment de Mathématiques, Université de Paris - Nord, 93430 Villetaneuse, France

C.E.A., Centre de Limeil (MA), B.P.27 94190 Villeneuve Saint Georges, France 